\title{
Interleukin-21 combined with ART reduces inflammation and viral reservoir in SIV-infected macaques
}

Luca Micci, ${ }^{1}$ Emily S. Ryan, ${ }^{1}$ Rémi Fromentin, ${ }^{2}$ Steven E. Bosinger, ${ }^{1,3}$ Justin L. Harper, ${ }^{1}$ Tianyu He, ${ }^{4}$ Sara Paganini, ${ }^{1}$ Kirk A. Easley, ${ }^{5}$ Ann Chahroudi, ${ }^{1,6}$ Clarisse Benne, ${ }^{7}$ Sanjeev Gumber, ${ }^{8,9}$ Colleen S. McGary, ${ }^{1}$ Kenneth A. Rogers, ${ }^{1}$ Claire Deleage, ${ }^{10}$ Carissa Lucero, ${ }^{10}$ Siddappa N. Byrareddy, ${ }^{9}$ Cristian Apetrei, ${ }^{4}$ Jacob D. Estes, ${ }^{10}$ Jeffrey D. Lifson, ${ }^{10}$ Michael Piatak Jr., ${ }^{10}$ Nicolas Chomont, ${ }^{2}$ Francois Villinger, ${ }^{1,9}$ Guido Silvestri, ${ }^{1,9}$ Jason M. Brenchley, ${ }^{11}$ and Mirko Paiardini ${ }^{1,9}$

'Division of Microbiology and Immunology, Yerkes National Primate Research Center (YNPRC), Emory University School of Medicine, Atlanta, Georgia, USA. ²Department of Microbiology, Infectiology, and Immunology, Université de Montréal, Faculty of Medicine, and Centre de Recherche du CHUM, Montreal, Quebec, Canada. ${ }^{3}$ Yerkes Nonhuman Primate Genomics Core, Emory University, Atlanta, Georgia, USA. “'Center for Vaccine Research, University of Pittsburgh, Pittsburgh, Pennsylvania, USA. ${ }^{5}$ Department of Biostatistics and Bioinformatics, Rollins School of Public Health, and ${ }^{6}$ Department of Pediatrics, Emory University School of Medicine, Atlanta, Georgia, USA. 'Department of Pathology, Case Western Reserve University, Cleveland, Ohio, USA. ${ }^{8}$ Division of Pathology, Yerkes National Primate Research Center, Atlanta, Georgia, USA. ${ }^{9}$ Department of Pathology and Laboratory Medicine, Emory University School of Medicine, Atlanta, Georgia, USA

${ }^{10}$ AIDS Cancer Virus Program, Frederick National Laboratory for Cancer Research, Leidos Biomedical Research Inc., Frederick, Maryland, USA.

"Laboratory of Molecular Microbiology, National Institute of Allergy and Infectious Diseases (NIAID), NIH, Bethesda, Maryland, USA.

Despite successful control of viremia, many HIV-infected individuals given antiretroviral therapy (ART) exhibit residual inflammation, which is associated with non-AIDS-related morbidity and mortality and may contribute to virus persistence during ART. Here, we investigated the effects of IL-21 administration on both inflammation and virus persistence in ARTtreated, SIV-infected rhesus macaques (RMs). Compared with SIV-infected animals only given ART, SIV-infected RMs given both ART and IL-21 showed improved restoration of intestinal Th17 and Th22 cells and a more effective reduction of immune activation in blood and intestinal mucosa, with the latter maintained through 8 months after ART interruption. Additionally, IL-21, in combination with ART, was associated with reduced levels of SIV RNA in plasma and decreased CD4+ T cell levels harboring replication-competent virus during ART. At the latest experimental time points, which were up to 8 months after ART interruption, plasma viremia and cell-associated SIV DNA levels remained substantially lower than those before ART initiation in IL-21-treated animals but not in controls. Together, these data suggest that IL-21 supplementation of ART reduces residual inflammation and virus persistence in a relevant model of lentiviral disease and warrants further investigation as a potential intervention for HIV infection.

\section{Introduction}

While combination antiretroviral therapy (ART) has dramatically improved the prognosis of HIV-infected individuals, a scalable treatment that can cure this infection is still not available. Residual disease in ART-treated HIV-infected individuals consists mainly of (a) persistent immune abnormalities, including inflammation and immune activation, limited $\mathrm{CD}^{+} \mathrm{T}$ cell reconstitution, and mucosal immune dysfunction (1), and (b) persistent reservoirs of latently infected cells that are not affected by ART and are responsible for the rapid rebound of virus replication upon ART discontinuation (2). Elevated levels of inflammation and immune activation that can persist even in ART-treated HIV-infected individuals with effective viral suppression have emerged as key correlates of morbidity and mortality from non-AIDS complications

Authorship note: Luca Micci and Emily S. Ryan contributed equally to this work. Michael Piatak Jr. is deceased.

Conflict of interest: The authors have declared that no conflict of interest exists. Submitted: February 6, 2015; Accepted: September 24, 2015.

Reference information: J Clin Invest. 2015;125(12):4497-4513. doi:10.1172/JCI81400. such as cardiovascular disease, neurocognitive dysfunction, and kidney and bone abnormalities, among others $(1,3)$. Furthermore, residual inflammation may contribute to HIV persistence during ART by several mechanisms, such as favoring de novo infection of activated "target" $\mathrm{CD} 4{ }^{+} \mathrm{T}$ cells that replenish the reservoir and upregulating the expression of immune checkpoint blockers that may limit the function of HIV-specific immune responses (1, 3, $4)$. Therefore, a vicious cycle in which inflammation, poor antiviral responses, and HIV persistence are intimately connected may occur in ART-treated HIV-infected individuals.

IL-21 is a pleiotropic cytokine that promotes the maintenance and functionality of Th17 cells (5-7), a subset of CD4 ${ }^{+} \mathrm{T}$ cells critical for mucosal immunity (8-14). Previously, we have shown that loss of intestinal IL-21-producing cells in the gut of SIV-infected rhesus macaques (RMs) is associated with the severity of Th17 cell depletion (15). Thus, reduced IL-21 availability may contribute to the loss of antimicrobial mucosal immunity and the induction and/or maintenance of mucosal immune dysfunction and associated pathological chronic immune activation during HIV infection. Consistent with this model, we showed that administration of a rhesus IL-21- 
IgFc fusion protein during acute SIV infection of RMs results in the preservation of intestinal Th17 cells, improved mucosal immune function, and reduced microbial translocation (16).

In the current study, we investigated the effects of exogenous IL-21 administration on residual immune activation and virus persistence in ART-treated, chronically SIV-infected RMs. Our findings, generated in a relevant primate model of lentiviral infection, indicate that IL-21 administration may have a significant beneficial effect on reducing residual inflammation and virus persistence during ART.

\section{Results}

Experimental design and antiretroviral therapy. Sixteen adult, Indianorigin RMs were infected i.v. with $30050 \%$ tissue culture infective dose $\left(\mathrm{TCID}_{50}\right)$ SIVmac $_{239}$. Beginning on day 60 after infection (day 60 p.i.), all animals were treated with a 5-drug antiretroviral regimen - PMPA (tenofovir), FTC (emcitrabine), raltegravir, and ritonavir-boosted darunavir - for 7 months (as indicated by the gray shading in Figure 1). Eight RMs (IL-21-treated) also received rhesus IL-21-IgFc fusion protein at $100 \mu \mathrm{g} / \mathrm{kg}$ (s.c.), which is a dosage comparable to that used in human clinical trials (17). The IL-21-treated RMs received 2 cycles of 6 weekly doses -1 at the time of ART initiation and 1 later on while on ART - plus 4 additional weekly doses at the time of ART interruption (orange arrows in Figure 1). The weekly dose regimen was selected on the basis of our previous study of IL-21 administration during acute SIV infection of RMs (16), as well as of our experience with in vivo administration of other cytokines in nonhuman primates (NHPs), in which weekly doses were better tolerated and showed a better response profile than did more frequent dosages (18-20). The remaining 8 RMs were treated only with ART and served as controls. Peripheral blood (PB), colorectal mucosa (RB), and lymph node (LN) biopsies were collected longitudinally and at necropsy (Figure 1A). Plasma viral load $\left(\log _{10}\right)$ before ART initiation was comparable between IL-21-treated ( $n=7$, animal ROc10 was excluded) and control $(n=8)$ RMs (4.98 \pm 0.40 vs. $5.11 \pm 0.40$, respectively; $P=0.8354)$. Both ART and IL-21 were well tolerated, without major clinical complications throughout the study. Animal ROc10 in the IL-21-treated group was lost on day 140 p.i. due to postsurgical complications of a LN biopsy. Thus, all analyses after day 135 p.i. include 7 IL-21treated RMs. Of note, ART was effective in suppressing viral replication, with all 16 RMs displaying plasma viremia levels below the limit of detection (LOD) of the assay (60 copies/ml) (Figure 1, $\mathrm{B}$ and $\mathrm{C}$ ). This level of suppression approaches the levels seen in ART-treated HIV-infected subjects, thus highlighting the validity of the model to test in vivo interventions targeting residual inflammation and virus persistence during ART. Clinical and immunogenetic characteristics of the 16 animals included in the study are summarized in Supplemental Table 1 (supplemental material available online with this article; doi:10.1172/JCI81400DS1).

Effects of IL-21 supplementation on blood and tissue $C D 4^{+} T$ cell levels. We next assessed the immunological impact of IL-21 supplementation of ART by measuring the relative frequencies and absolute numbers of $\mathrm{CD}^{+}$and $\mathrm{CD}^{+} \mathrm{T}$ cells in the sampled anatomical sites of our cohort of SIV-infected, ART-treated RMs. As expected, we found that ART was effective at increasing $\mathrm{CD} 4^{+} \mathrm{T}$ cell numbers in $\mathrm{PB}, \mathrm{RB}$, and $\mathrm{LN}$, although the reconstitution was still incomplete after 7 months on ART as compared with preinfection cell numbers, especially in RB (Figure 1, D-G). The numbers of total $\mathrm{CD}^{+} \mathrm{T}$ cells as well as key central memory (CM) $\left(\mathrm{CD} 28^{+}\right.$ $\left.\mathrm{CD} 5^{+} \mathrm{CCR} 7^{+}\right)$and effector memory (EM) $\left(\mathrm{CD} 28^{+} \mathrm{CD} 95^{+} \mathrm{CCR} 7^{-}\right)$ subsets (Supplemental Figure 1) in PB (Figure 1, D and E), RB (Figure 1F), and LN (Figure 1G) were comparable between the 2 groups (i.e., ART alone versus ART plus IL-21) at all experimental time points. Thus, while ART was effective at suppressing virus replication and improved $\mathrm{CD} 4^{+} \mathrm{T}$ cell restoration, supplementation with IL-21 did not improve the reconstitution of CD4 ${ }^{+} \mathrm{T}$ cells in either blood or tissues.

Effects of IL-21 supplementation on blood and LN CD $8^{+} T$ cell and $B$ cell levels. We next assessed the effects of IL-21 on total CD8 ${ }^{+} \mathrm{T}$ cell and B cell levels as well as on their main differentiation subsets in PB and LN. The levels of blood (percentages and counts), $\mathrm{LN}$ (percentages), and RB (percentages) $\mathrm{CD}^{+} \mathrm{T}$ cells (Supplemental Figure 2) and their subsets (data not shown) were overall very similar in IL-21-treated and control RMs, with no significant differences between the 2 groups at any single experimental time point. Previous studies showed that IL-21 stimulates the production of cytotoxic molecules in CD8 ${ }^{+} \mathrm{T}$ cells in humans in vitro (21) and in acutely SIV-infected RMs in vivo (16). Hence, we sought to determine by flow cytometry the longitudinal expression of intracellular perforin, granzyme $\mathrm{B}(\mathrm{GrB})$, and T-bet in blood and $\mathrm{LN} \mathrm{CD} 8^{+} \mathrm{T}$ cells. No significant differences were noted in the fraction of $\mathrm{CD}^{+}$ $\mathrm{T}$ cells expressing any of these markers (Supplemental Figure 3), or a combination of them (data not shown), between IL-21-treated and control RMs. Similar to $\mathrm{CD}^{+} \mathrm{T}$ cells, the percentages of blood and $\mathrm{LN} \mathrm{B}$ cells $\left(\mathrm{CD} 2 \mathrm{O}^{+}\right)$, as well as their naive $\left(\mathrm{CD} 21^{+} \mathrm{CD} 27^{-}\right)$, resting memory $\left(\mathrm{CD} 21^{+} \mathrm{CD} 27^{+}\right)$, activated memory $\left(\mathrm{CD} 21^{-} \mathrm{CD} 27^{+}\right)$, and tissue memory (CD21- $\mathrm{CD} 27^{-}$) subsets (22), were also not affected by IL-21 treatment when assessed by flow cytometry (Supplemental Figure 4). Interestingly, IHC analyses of LN biopsies showed that IL-21-treated animals had significantly higher levels of B cells in the medulla when compared with controls (Supplemental Figure 5), thus suggesting that increased availability of IL-21 in the LN (as determined by IHC staining, data not shown) can impact the anatomic distribution of B cells during SIV infection.

IL-21 increases intestinal Th17 and Th22 cell levels in ARTtreated, SIV-infected RMs. To assess the effects of IL-21 treatment on mucosal immunity, we next measured the levels of intestinal Th17 and Th22 cells, identified as CD $4^{+} \mathrm{T}$ cells producing IL-17 and IL-22, respectively, after brief in vitro stimulation with PMA and ionomycin. Representative staining of IL-17 (Figure 2A) and IL-22 (Figure 2B) within the intestinal CD $4^{+} \mathrm{T}$ cells is shown in 1 IL-21-treated and 1 control RM before infection (day 20), before ART (day 58 p.i.), and at 3 time points when on ART. The reconstitution of intestinal Th17 (Figure 2C) and Th22 (Figure 2D) cells was very limited in the RMs treated with ART alone, while IL-21 supplementation was associated with a faster and more pronounced reconstitution of both cell types. Indeed, IL-21-treated RMs showed significantly higher frequencies of Th17 and Th22 cells compared with frequencies in control animals at day 85 p.i. (day 25 on ART; Th17: $P=0.0003$, Th22: $P=0.0096$ ), day 135 p.i. (Th17: $P=0.0029$; Th22: $P=0.0148)$, and day 203 p.i. (Th17: $P=0.0052)$ (Figure 2, C and D). Remarkably, in IL-21-treated animals, the frequencies of Th17 cells on days 85 and 135 p.i. were 

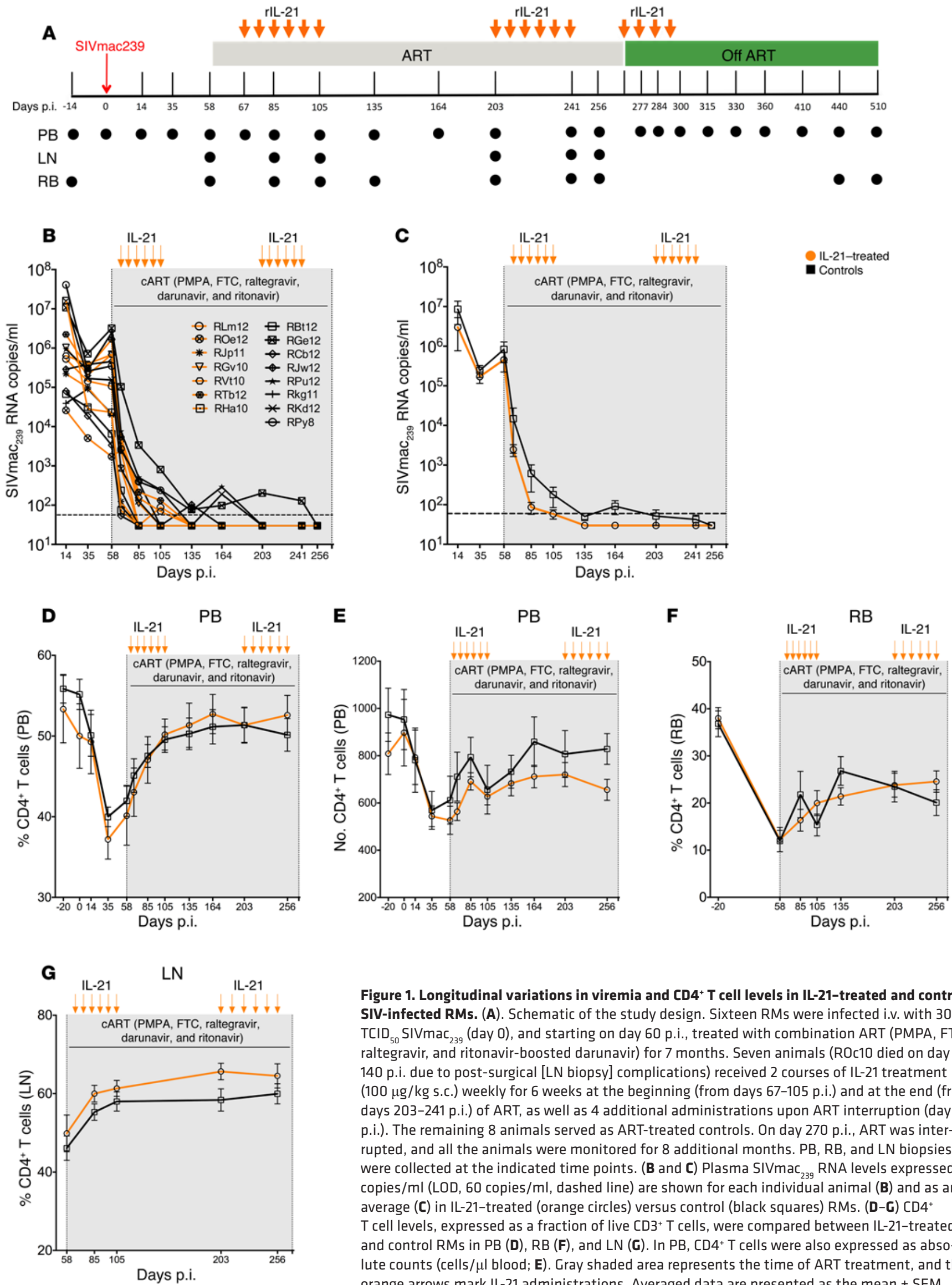

Figure 1. Longitudinal variations in viremia and CD4 ${ }^{+} \mathrm{T}$ cell levels in IL-21-treated and control SIV-infected RMs. (A). Schematic of the study design. Sixteen RMs were infected i.v. with 300 $\mathrm{TCID}_{50} \mathrm{SIVmac}_{239}$ (day 0 ), and starting on day 60 p.i., treated with combination ART (PMPA, FTC, raltegravir, and ritonavir-boosted darunavir) for 7 months. Seven animals (ROc10 died on day 140 p.i. due to post-surgical [LN biopsy] complications) received 2 courses of IL-21 treatment (100 $\mu \mathrm{g} / \mathrm{kg}$ s.c.) weekly for 6 weeks at the beginning (from days 67-105 p.i.) and at the end (from days 203-241 p.i.) of ART, as well as 4 additional administrations upon ART interruption (day 271 p.i.). The remaining 8 animals served as ART-treated controls. On day 270 p.i., ART was interrupted, and all the animals were monitored for 8 additional months. PB, RB, and LN biopsies were collected at the indicated time points. (B and C) Plasma SIVmac ${ }_{239}$ RNA levels expressed as copies/ml (LOD, 60 copies/ml, dashed line) are shown for each individual animal (B) and as an average (C) in IL-21-treated (orange circles) versus control (black squares) RMs. (D-G) CD4+ $T$ cell levels, expressed as a fraction of live $\mathrm{CD3}^{+} \mathrm{T}$ cells, were compared between IL-21-treated and control RMs in PB (D), RB (F), and LN (G). In PB, CD4+ T cells were also expressed as absolute counts (cells/ $\mu$ l blood; E). Gray shaded area represents the time of ART treatment, and the orange arrows mark IL-21 administrations. Averaged data are presented as the mean \pm SEM. CART, combination ART. 
A
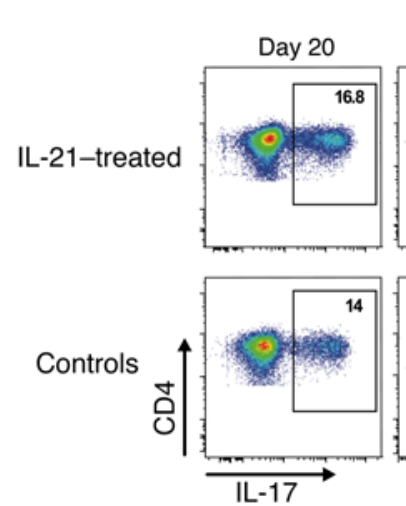

B

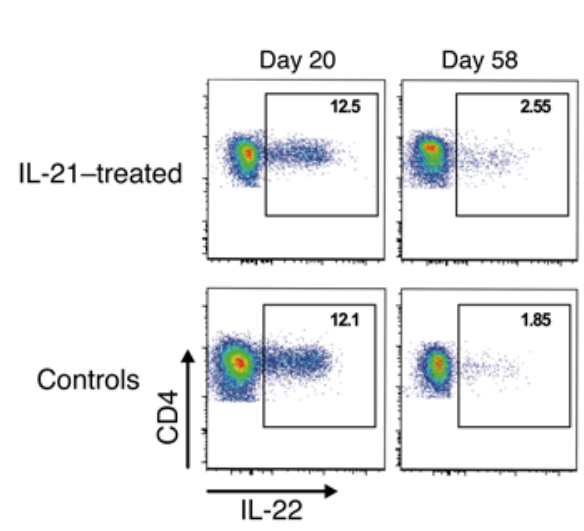

\section{E}

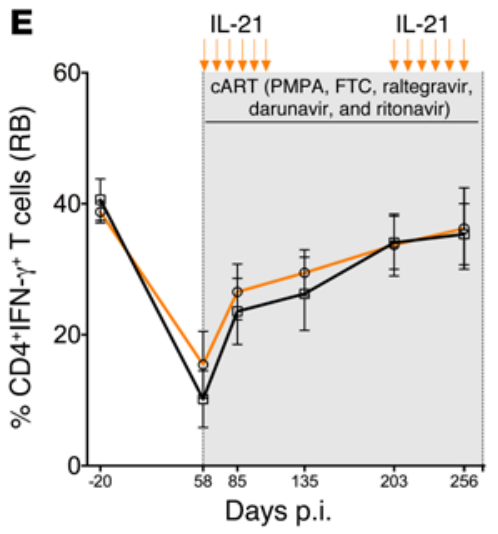

G

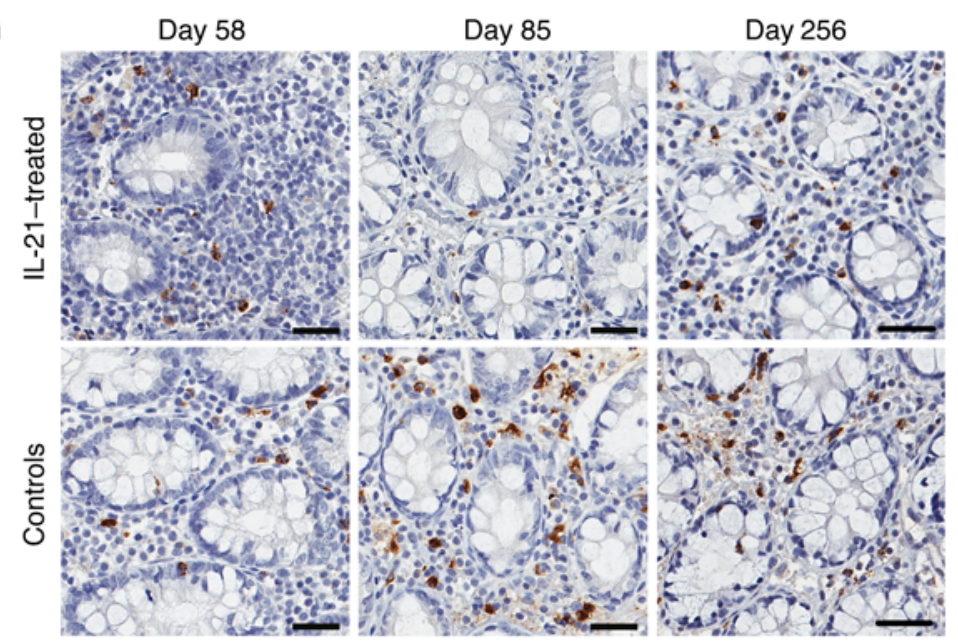

On ART

Day 85 p.i. Day 135 p.i. Day 256 p.i.
(day 25 on ART) (day 75 on ART) (day 200 on ART)
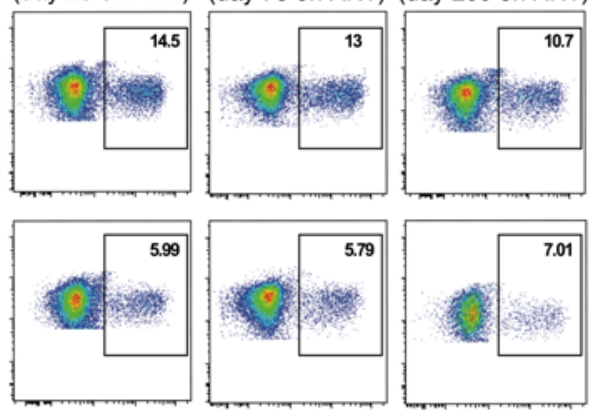

On ART

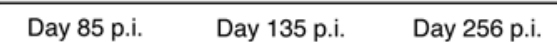
(day 25 on ART) (day 75 on ART) (day 200 on ART)
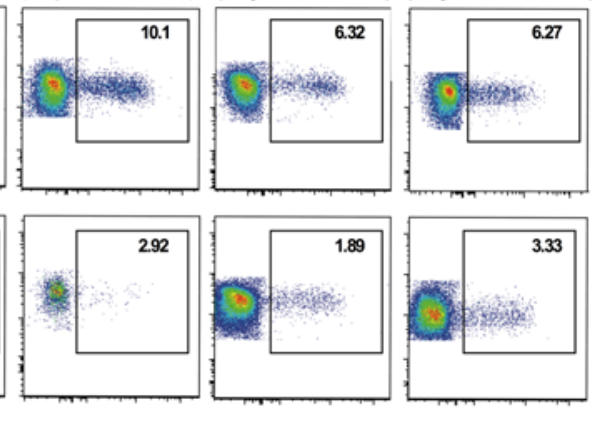

\section{$\mathbf{F}$}

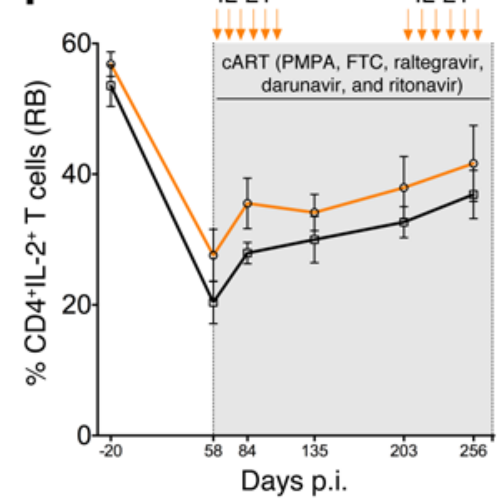

C

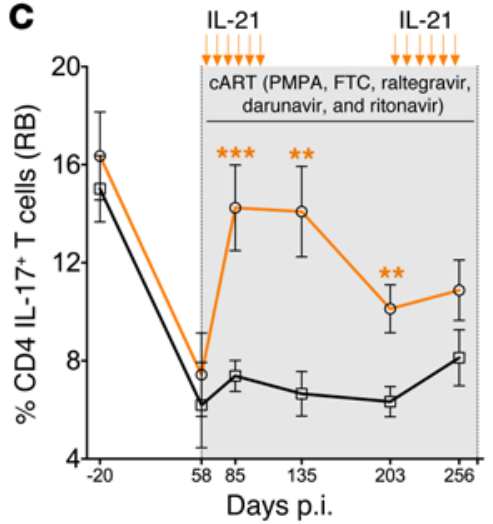

D

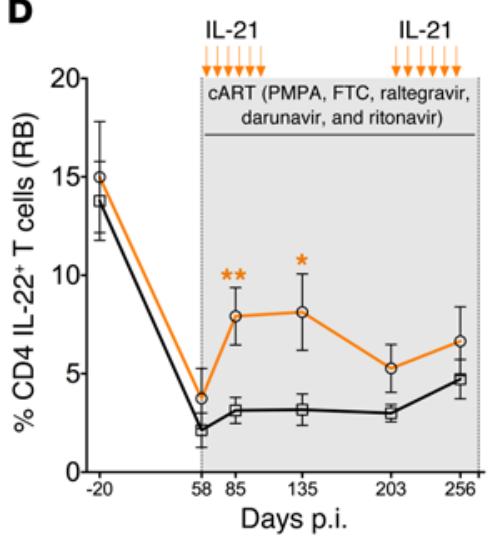

IL-21-treated Controls
H

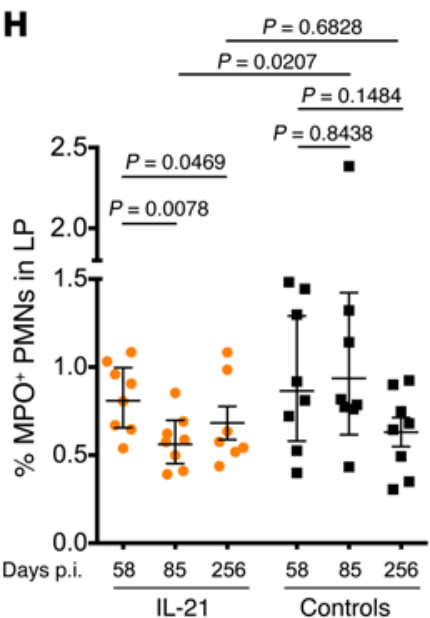


Figure 2. IL-21 supplementation improves mucosal immunity in ARTtreated, SIV-infected RMs. (A and B) Representative staining for IL-17 (A) and IL-22 (B) within intestinal CD4 ${ }^{+}$T cells in IL-21-treated (top plots) and control (bottom plots) RMs before infection (day -20), after infection but before ART and IL-21 initiation (day 58 p.i.), and at 3 different experimental time points on ART (days 25, 75, and 200). (C and F) Longitudinal assessment of the frequency of intestinal CD4+ $T$ cells producing IL-17 (C), IL-22 (D), IFN- $\gamma(\mathbf{E})$, and IL-2 (F) in IL-21-treated $(n=7)$ and control $(n=8)$ RMs. ( $\mathbf{G}$ and $\mathbf{H}$ ) Rectal mucosa tissues were stained for MPO (brown) as a marker for PMNs. (G) Representative MPO staining (original magnification, $\times 200$ ) in $1 \mathrm{IL}-21$-treated RM (top panels) and 1 control RM (bottom panels) before ART (day 58 p.i.) and at day 25 (day 85 p.i.) and day 200 (day 256 p.i.) on ART. (H) Random, high-powered $(\times 400)$ images of gut LP were taken, and the percentage of area staining for MPO was determined in IL-21-treated and control RMs. Shaded area represents the time of ART treatment, and orange arrows represent IL-21 administrations. Averaged data are presented as the mean \pm SEM. Repeated-measures analyses were performed with a means model (SAS PROC MIXED, version 9) to generate statistical outcomes. ${ }^{*} P<0.05 ;{ }^{* *} P<0.01 ;{ }^{* *} P<0.001$.

comparable to their preinfection levels. IL-21-treated animals also had significantly higher levels of $\mathrm{CD} 4^{+} \mathrm{IL}-17^{+} \mathrm{IL}-22^{+} \mathrm{T}$ cells on days 85 and 135 p.i. when compared levels in control animals (Supplemental Figure 6). The effects of IL-21 were specific for the Th17 and Th22 subsets, as the frequencies of intestinal CD $4^{+}$ IFN- $\gamma^{+}$(Figure 2E) and CD $4{ }^{+} \mathrm{IL}_{-} 2^{+}$(Figure 2F) T cells were indistinguishable between the 2 groups and were more pronounced when IL-21 was administered earlier during ART (first cycle of 6 doses), as compared with the frequencies detected during late ART (second cycle). During SIV infection of RMs, polymorphonuclear neutrophil (PMN) activation leads to accumulation of myeloperoxidase-positive $\left(\mathrm{MPO}^{+}\right)$PMNs adjacent to epithelial lesions, with the extent of MPO expression strongly correlating with the degree of intestinal epithelial barrier dysfunction (10). To assess the impact of IL-21 supplementation on this marker of mucosal immune dysfunction, we performed IHC to measure PMN infiltration in colorectal biopsy tissues from IL-21-treated and control animals before ART (day 58 p.i.) and at early (day 85 p.i.) and late (day 256 p.i.) experimental time points on ART (Figure $2 \mathrm{G}$ ). In IL-21-treated RMs, but not in controls, the levels of $\mathrm{MPO}^{+} \mathrm{PMNs}$ in the lamina propria (LP), in both early and late ART, were significantly lower than the levels observed on day 58 p.i. $(P=0.0078$ and $P=0.0469$, respectively; Figure $2 \mathrm{H})$ and were thus consistent with a decrease of intestinal inflammation and, possibly, improved mucosal integrity following IL-21 treatment. Furthermore, during early ART, $\mathrm{MPO}^{+} \mathrm{PMN}$ levels were significantly lower in IL-21-treated RMs as compared with levels in controls $(P=0.0207$; Figure $2 \mathrm{H})$, consistent with the higher numbers of Th17 and Th22 cells at the same experimental point. At the later time point (day 256 p.i.), ART alone became more effective at reducing MPO expression in PMNs, resulting in comparable MPO levels in the 2 groups $(P=0.6828$; Figure $2 \mathrm{H})$, which is consistent with the reduced differences in Th17 and Th22 cell levels at the same experimental time point.

Taken together, these data indicate that IL-21 administration improves the reconstitution of intestinal Th17 and Th22 cells and reduced intestinal inflammation in ART-treated, SIV-infected RMs.

IL-21 treatment reduces residual $T$ cell activation and proliferation in ART-treated, SIV-infected RMs. Preferential loss of intestinal
Th17 and Th22 cells is a key mechanism of mucosal immune dysfunction and chronic immune activation during pathogenic HIV/ SIV infections $(8,13,14,23,24)$. To investigate whether the higher IL-21-induced numbers of intestinal Th17 and Th22 cells were associated with reductions in $\mathrm{T}$ cell activation and/or proliferation when on ART, we next examined the frequencies of $\mathrm{CD}^{+}$and $\mathrm{CD}^{+} \mathrm{T}$ cells expressing the activation markers HLA-DR and CD38 and/or the proliferation marker Ki-67. We found that IL-21-treated RMs showed a more rapid - i.e., already evident at day 25 on ART (day 85 p.i.) - and more pronounced reduction of activated memory $\left(\mathrm{CD} 28^{+} \mathrm{CD} 5^{+}\right) \mathrm{CD}^{+}$and $\mathrm{CD} 8^{+} \mathrm{T}$ cells in $\mathrm{PB}$ (Figure $3, \mathrm{~A}$ and D) and RB (Figure 3, G and J) up to day 200 on ART (day 256 p.i.), and a similar observation was made in the LN (Supplemental Figure 7, A and B). Furthermore, IL-21-treated RMs showed a more rapid and pronounced reduction in both memory $\mathrm{CD} 4^{+}$and $\mathrm{CD} 8^{+}$ T cell proliferation levels in rectum $(P \leq 0.01$; Supplemental Figure $8, \mathrm{~A}$ and $\mathrm{B}$ ) and blood ( $P \leq 0.01$; data not shown). Interestingly, in blood (Figure 3, B and C), but not in RB (Figure 3, H and I), the reduction of immune activation was more pronounced for the key population of $\mathrm{CM} \mathrm{CD} 4^{+} \mathrm{T}$ cells compared with that observed in EM CD $4^{+} \mathrm{T}$ cells. This was specific for $\mathrm{CD} 4^{+}$lymphocytes, since IL-21-treated RMs have lower levels of activation in both blood (Figure 3, E and F) and RB (Figure 3, $\mathrm{K}$ and $\mathrm{L}$ ) $\mathrm{CM}$ and $\mathrm{EM} \mathrm{CD} 8^{+} \mathrm{T}$ cells. Finally, plasma levels of soluble inflammatory markers such as IP-10, which are associated with HIV morbidity and mortality (days 143 and 200 on ART; $P=0.0401$ ) and C-reactive protein (CRP) (day 75 on ART; $P=0.0323$ ), were also significantly lower in IL-21-treated RMs as compared with levels in ART-only controls (Supplemental Figure 8, C and D).

These data indicate that IL-21 supplementation in ARTtreated, SIV-infected RMs is associated with a more rapid and pronounced reduction of residual immune activation levels.

$I L-21$ treatment upregulates the expression of genes regulating antimicrobial immunity in ART-treated, SIV-infected RMs. We further explored the effect of IL-21 supplementation on ARTtreated, SIV-infected RMs by comparing the whole transcriptome, as assessed by RNA sequencing (RNA-seq), in peripheral blood mononuclear cells (PBMCs) collected before ART (day 58 p.i.) and at 2 experimental time points on ART (days 50 and 200 on ART) in our 2 groups of animals. Changes in the expression of a large number of shared genes were observed in both IL-21-treated and control samples as a result of ART-induced viral suppression. Differential regulation between the 2 groups of RMs was observed in 53 genes at day 50 and in 42 genes at day 200 on ART $(Q<0.05)$ (Figure 4, A and E). Specifically, and consistent with the increased levels of Th17 and Th22 cell subsets (Figure 2), numerous genes associated with antimicrobial immunity were significantly upregulated in IL-21-treated animals as compared with controls at day 50 p.i. (Figure $4 \mathrm{~B}$ ). Among these, $L T F$, whose protein product is lactotransferrin, a major iron-binding protein with a broad range of antimicrobial and antiinflammatory functions (25), showed a 29-fold upregulation. Of note, lactotransferrin plays a key role in immunity against Candida albicans, an important opportunistic pathogen and cause of morbidity and mortality in HIV-infected subjects (26) and against which Th17 cells are highly specialized (27). LCN2 (lipocalin 2) and DEFA1 (defensin $\alpha 1$ ), both of which participate in neutrophil-mediated antimicrobial immunity, were 
Controls
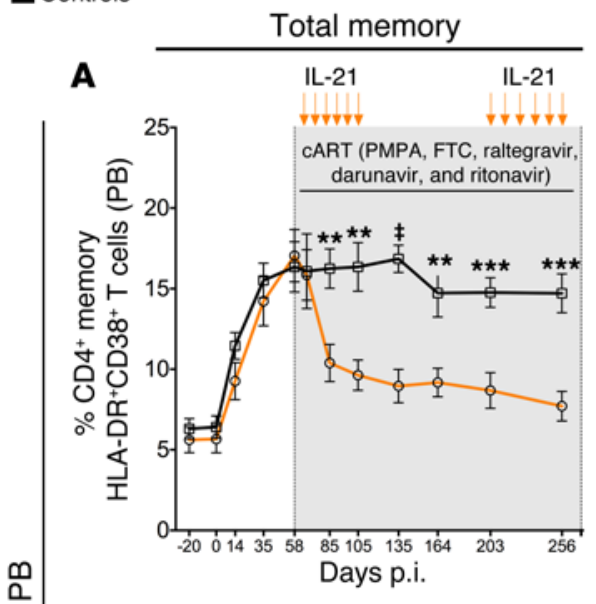

D

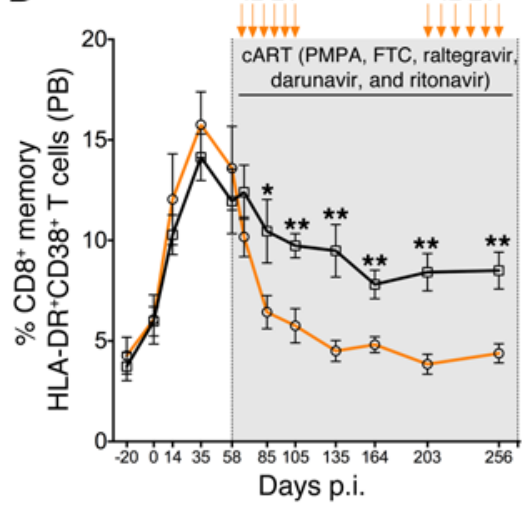

G

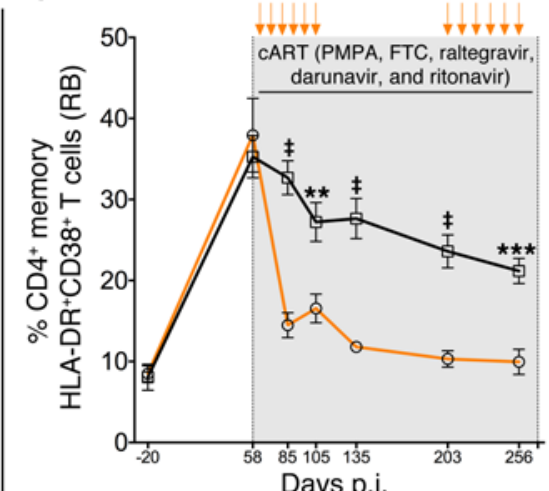

$\stackrel{m}{\widetilde{x}}$

J

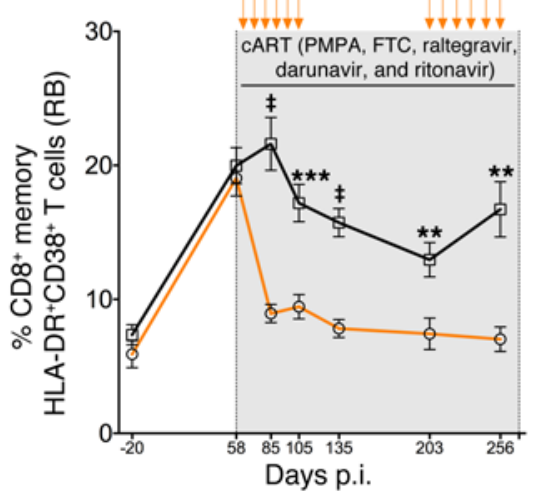

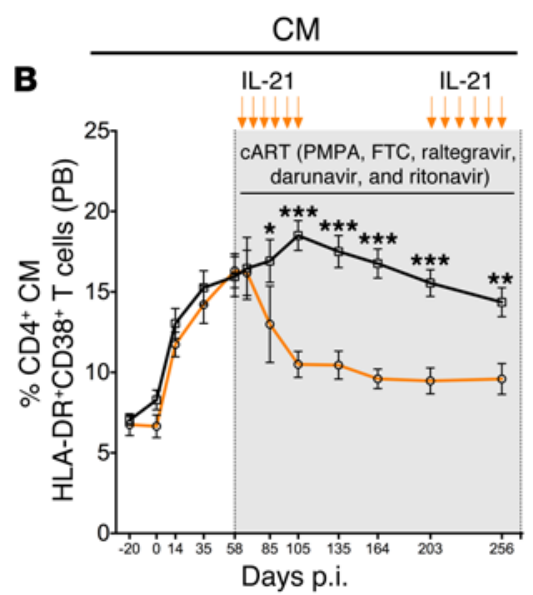

E

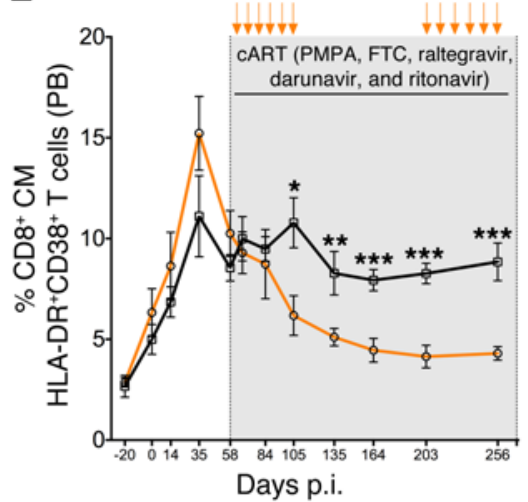

H

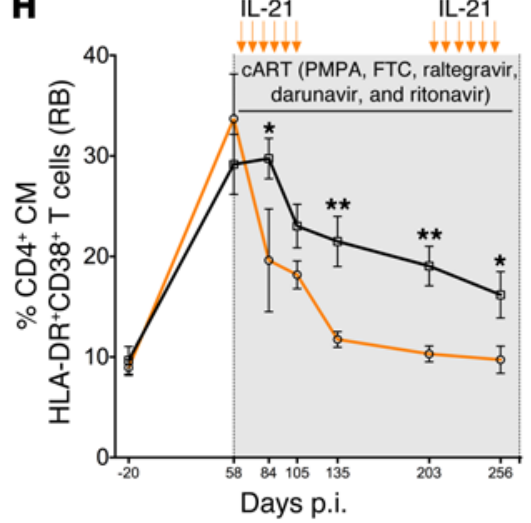

K

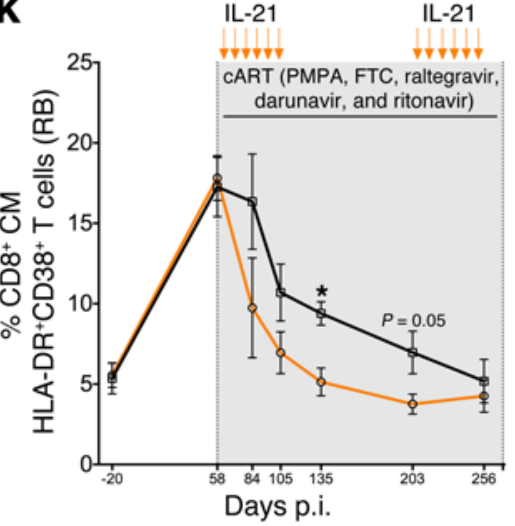

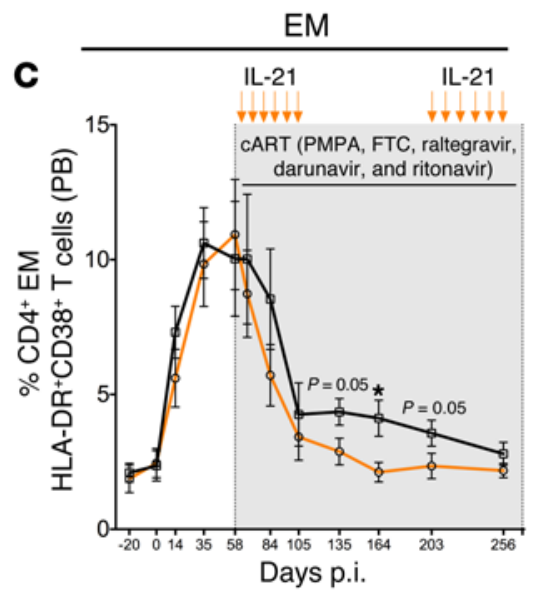

F

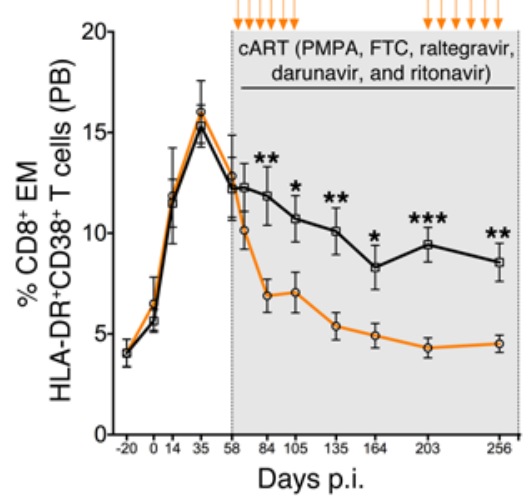

I

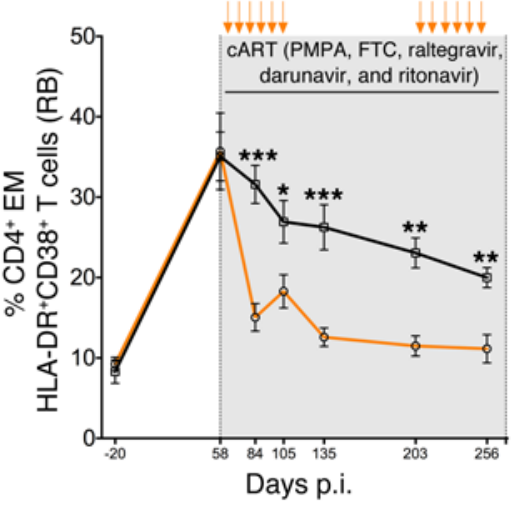

L

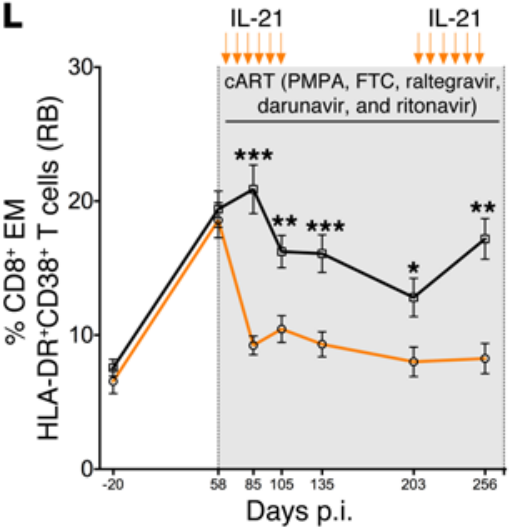


Figure 3. IL-21 supplementation limits residual T cell activation in ARTtreated, SIV-infected RMs. (A-F) Longitudinal assessment of the activation levels of total memory (A and $\mathbf{D}), \mathrm{CM}$ (B and $\mathbf{E})$, and EM (C and $\mathbf{F}) \mathrm{CD}^{+}(\mathbf{A}-\mathbf{C})$ and $\mathrm{CD}^{+}(\mathbf{D}-\mathbf{F}) \mathrm{T}$ cells in blood (PB). (G-L) Longitudinal assessment of the activation levels of total memory ( $\mathbf{G}$ and $\mathbf{J}), \mathbf{C M}(\mathbf{H}$ and $\mathbf{K})$, and EM (I and $\mathbf{L}$ ) $\mathrm{CD}^{+}(\mathbf{G}-\mathbf{I})$ and $\mathrm{CD} 8^{+}(\mathrm{J}-\mathrm{L}) \mathrm{T}$ cells in colorectum (RB). T cell activation was assessed by dual expression of the immune activation markers HLA-DR and CD38. IL-21-treated animals $(n=7)$ are indicated by orange circles and controls $(n=8)$ by black squares. Shaded area represents the time of ART treatment, and orange arrows represent IL-21 administrations. Averaged data are presented as the mean \pm SEM. Repeated-measures analyses were performed with a means model (SAS PROC MIXED, version 9) to generate statistical outcomes. ${ }^{*} P<0.05 ;{ }^{* *} P<0.01 ;{ }^{* *} P<0.001 ;{ }^{\ddagger} P<0.0001$.

upregulated 12-fold in IL-21-treated, SIV-infected RMs. Importantly, lipocalin 2 levels were found to be reduced in HIV infection (28). Several genes related to antimicrobial immunity were still upregulated in IL-21-treated RMs versus control RMs at the latest experimental time point on ART (Figure $4 \mathrm{~F}$ ). In addition to the above-mentioned $L T F$ gene, those specifically upregulated at day 200 on ART in the IL-21 treatment group included MUC4 (mucin 4, 2.2-fold increase), a major constituent of mucus membranes with a key role in the protection of epithelial mucosa (29); PF4 (platelet factor 4, 1.4-fold increase), which plays a role in wound repair, inflammation, and mucosal immunity (30); and TREM1 (triggering receptor expressed on myeloid cells 1, 1.4-fold increase), which participates in myeloid inflammatory responses to bacterial and fungal infections (31). Additionally, IL-21-treated RMs showed a 1.5-fold increase in PLK3 (polo kinase 3) (Figure 4F), which codes for a protein that regulates cell-cycle progression and has been shown to be downregulated in SIV-infected RMs that progress to AIDS but not in natural SIV hosts that remain AIDS free, such as the sooty mangabeys (SMs) (32). Furthermore, we found significant associations between the expression of several transcripts, which significantly increased with IL-21 treatment, and markers of T cell immune activation and SIV persistence (Supplemental Table 2). Collectively, our data are consistent with the hypothesis that the main mechanism by which IL-21 limits residual inflammation in ART-treated, SIV-infected RMs is a Th17/Th22 cell-dependent improvement in mucosal immunity. To further investigate this pathway, we used gene set enrichment analysis (GSEA) to test the enrichment of gene sets related to IL-21, IL-17, and IL-22 signaling and found that IL-21-treated RMs were enriched in these gene sets at day 50 p.i. $(P=0.047$; Figure $4, C$ and $D)$ and at day 200 on ART $(P=0.20$; Figure $4, \mathrm{G}$ and $\mathrm{H})$. Genes belonging to the JAK/STAT pathway, one of the major signaling routes for IL-21, IL-17, and IL-22, were significantly enriched in the IL-21-treated RMs (Supplemental Figure 9, A and B). Finally, a trend toward enrichment in the expression of IFN-stimulated genes (ISGs) was observed in IL-21-treated RMs at the earlier experimental time point, but at the later time point in controls (i.e., ART only) (Supplemental Figure 9, C and D), a finding that was thus compatible with the triggering of an early antimicrobial response in IL-21-treated RMs, which was then downregulated concomitantly with the reduced levels of $\mathrm{T}$ cell immune activation.

In summary, IL-21 administration in ART-treated, SIVinfected RMs induces a transcriptional profile associated with active antimicrobial immune responses and improved Th17 and Th22 cell homeostasis.
IL-21 treatment reduces viral persistence in ART-treated, SIV-infected $R M s$. Recent studies have linked residual immune activation to HIV persistence (33-35). Thus, we sought to determine whether the IL-21-induced reduction of immune activation and inflammation impacted the level of virus persistence during ART by measuring plasma levels of SIV RNA using an ultrasensitive assay (LOD, 3 copies of SIV RNA $/ \mathrm{ml}$ ). As shown in Figure 5, we found that IL-21treated RMs showed a progressive reduction in plasma SIV RNA levels compared with ART-only controls, with the fraction of RMs with an undetectable viral load remaining stable between days 75 and 200 on ART in controls (3 of 8 animals; 37.5\%) but gradually increasing from $28.5 \%$ to $85.7 \%$ of animals in the IL-21-treated group over time $(P=0.03$, test for interaction between time on study and treatment group; Figure $5 \mathrm{~A}$ ). This trend resulted in the levels of residual viremia being significantly lower in IL-21-treated RMs compared with controls by the end of the 200 days on ART $(P=0.0203)$. We next measured the frequency of cells harboring SIV DNA in the colorectum at 2 experimental time points on ART. Consistent with the data obtained for residual viremia, the reduction of SIV DNA levels (copies per $10^{8}$ cell equivalents) in RB biopsies between days 50 and 200 on ART was more pronounced in IL-21-treated RMs $(P=0.0156)$ than in controls $(P=0.1484)$ (Figure 5B) and therefore resulted in lower levels of SIV DNA in treated animals than in controls at day 200 on ART $(P=0.0607)$. The possibility of a link between immune activation and SIV persistence is supported by the observation that the frequencies of intestinal CD8 ${ }^{+} \mathrm{HLA}-\mathrm{DR}^{+} \mathrm{CD} 38^{+} \mathrm{T}$ cells at day $50(r=0.6368$; $P=0.0107)$ and day $200(r=0.6314 ; P=0.0116)$ on ART positively correlated with SIV DNA content at day 200 on ART (Figure 5C). In addition, the frequency of $\mathrm{CD} 4^{+} \mathrm{T}$ cells expressing PD1, a subset of cells enriched in HIV reservoirs (35), was also significantly lower in RB biopsies of IL-21-treated RMs than in controls at day 200 on ART (Supplemental Figure 10A). Of note, plasma viremia at ART initiation correlated significantly with SIV DNA contents in blood CD $4^{+}$T cells $(r=0.7821 ; P=0.0009)$ and trended toward significance with SIV DNA copies in RB $(r=0.5071 ; P=0.0562)$ at day 200 on ART, but did not correlate with the frequencies of intestinal CD8 ${ }^{+} \mathrm{HLA}-\mathrm{DR}^{+} \mathrm{CD} 38^{+} \mathrm{T}$ cells at day 50 or day 200 on ART (Supplemental Table 3).

Finally, we measured the levels of $\mathrm{CD} 4^{+} \mathrm{T}$ cells harboring replication-competent virus using a modified quantitative viral outgrowth assay (QVOA) previously described for SIV-infected RMs $(36,37)$. Highly purified LN CD4 ${ }^{+} \mathrm{T}$ cells were isolated from 4 IL-21-treated and 4 control RMs at the last experimental time point on ART (QVOA requires a large number of cells, thus we were limited to doing this assay only in animals with a large number of cryopreserved cells). Purified $\mathrm{CD} 4^{+} \mathrm{T}$ cells were cocultured with the CEMx174 cell line, and frequencies of cells expressing p27 (see Figure 6A showing a representative staining), as well as levels of SIV RNA released in the supernatant, were quantified on days 9, 16, and 25 after coculture. Remarkably, all 4 IL-21-treated RMs showed levels of p27 expression and SIV RNA in supernatant that were significantly lower than those in controls at all tested experimental time points (Figure 6, B and C). As a result, the frequencies of latently infected cells, expressed as infectious units per million (IUPM) CD4 ${ }^{+} \mathrm{T}$ cells, were significantly lower in IL-21treated RMs than in controls (Figure 6D). 
A

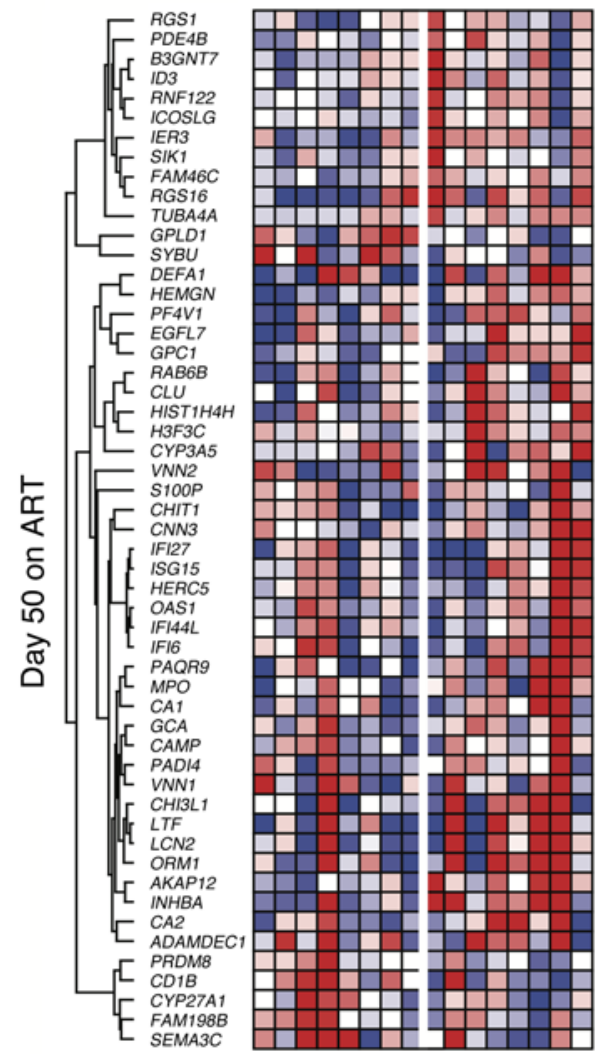

E

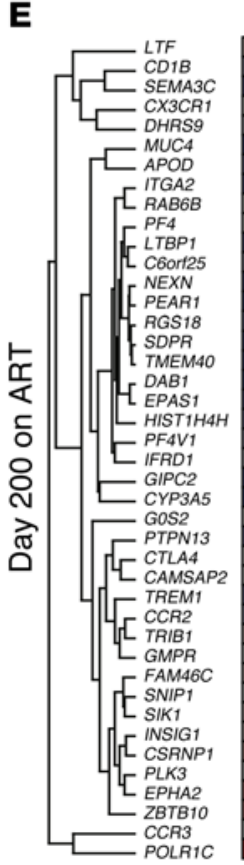

Control
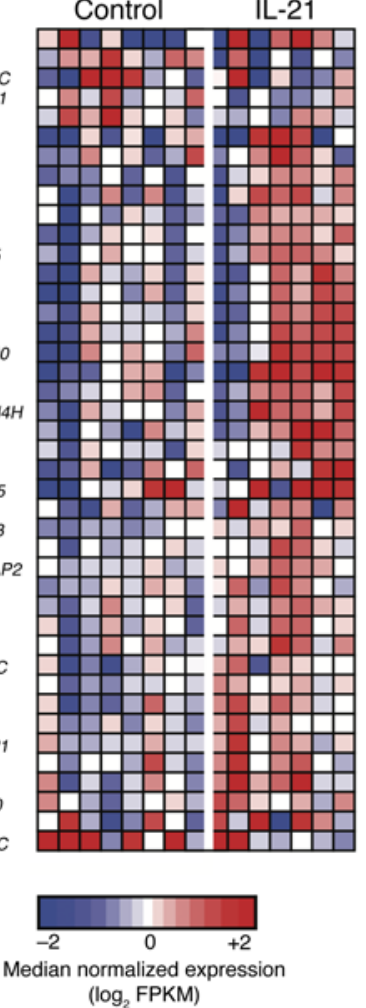

B
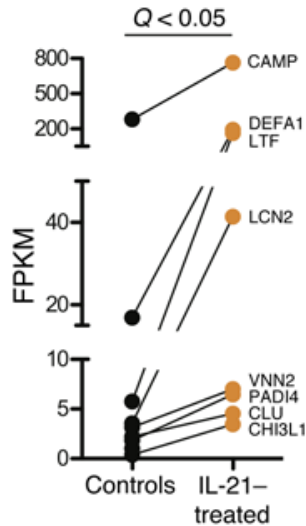

C

IL-21-, IL-17-, and IL-22-related genes

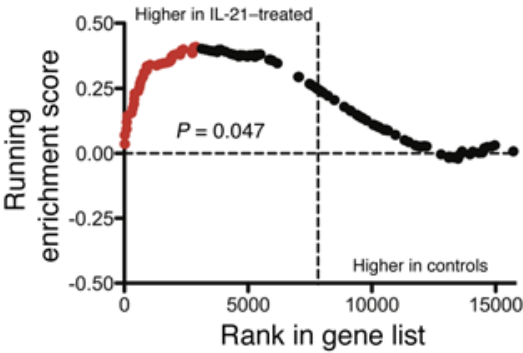

$\mathbf{F}$

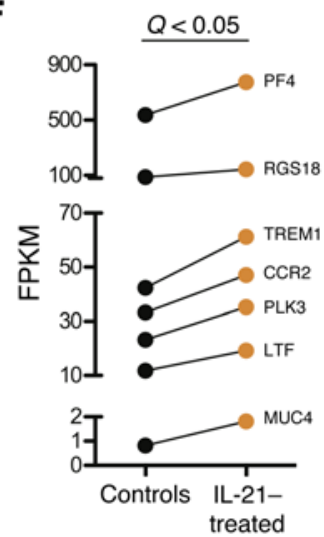

G

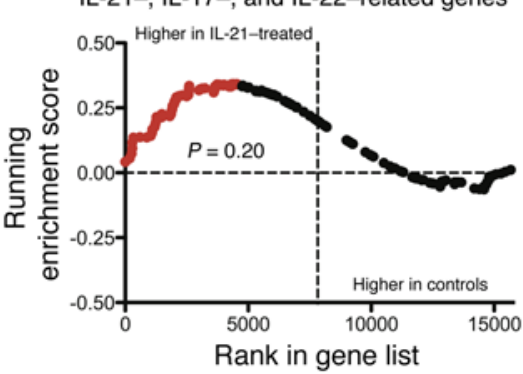

D

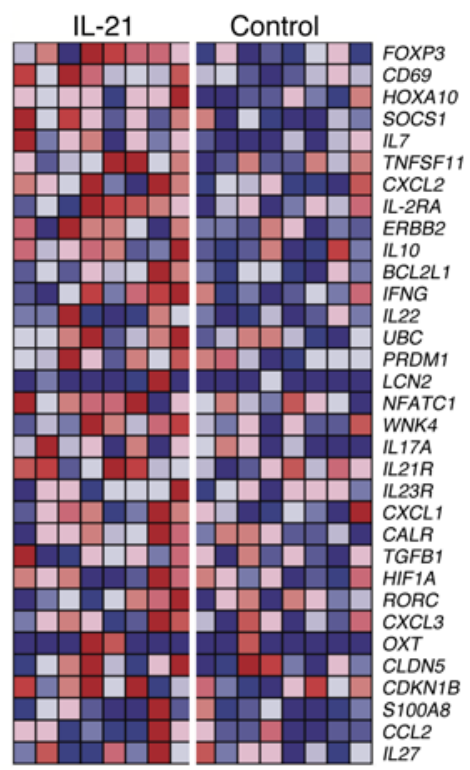

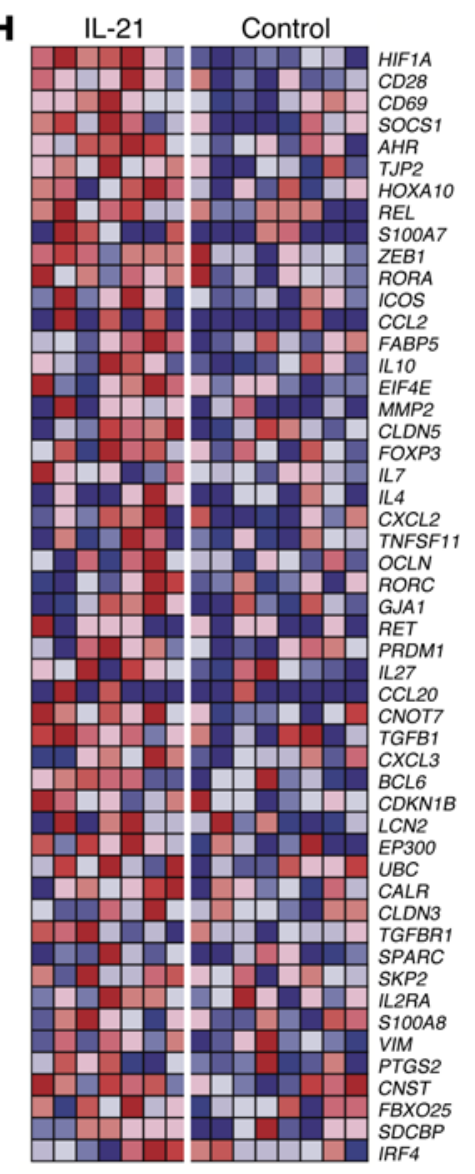


Figure 4. IL-21 supplementation upregulates the expression of genes regulating antimicrobial immunity in PBMCs from ART-treated, SIV-infected RMs. (A and E) Global heatmaps of genes whose expression levels were significantly different $(\underline{0}<0.05)$ between IL-21-treated $(n=7)$ and control $(n=8)$ RMs at day $50(\mathbf{A})$ or day 200 (E) on ART. The specific genes are indicated on the left side of the heatmaps. (B and F) FPKM values for the selected genes of interest were compared between IL-21-treated (orange circles) and control (black squares) RMs at day 50 (B) and day 200 (F) on ART. (C and G) Gene ranking for IL-21-, IL-17-, and IL-22-related genes at day 50 (C) and day 200 (G) on ART. Dots represent individual genes plotted according to their ranking ( $x$ axis) and their addition to the cumulative score ( $y$ axis). $P$ values below 0.25 were considered significant according to GSEA guidelines. Leading genes with higher enrichment scores in IL-21treated RMs (as compared with controls) are highlighted in red and shown as heatmaps in $\mathbf{D}$ and $\mathbf{H}$. Statistical analyses were performed with the Partek Genomics Suite, version 6.6, and the GSEA tool.

Along with those shown in Figure 3, these results indicate that IL-21 supplementation of ART limits both inflammation and viral persistence in SIV-infected RMs and support the possibility of a direct link between mucosal immunity, inflammation, and HIV persistence. Supporting this molecular link, SIV DNA content in purified blood $\mathrm{CD}^{+} \mathrm{T}$ cells at the latest time point on ART (day 256 p.i.) correlated (a) negatively with the levels of intestinal Th17 $(r=-0.7632 ; P=0.0013$; Supplemental Figure 11A) and Th22 cells $(r=-0.6429 ; P=0.0116$; Supplemental Figure 11C) before ART (day 58 p.i.); (b) negatively with the levels of intestinal Th17 cells on ART (day 105 p.i.; $r=-0.5898 ; P=0.0223$; Supplemental Figure 11B); and (c) positively with the levels of intestinal CD $4^{+} \mathrm{Ki}-67^{+} \mathrm{T}$ cells on ART (day 84 p.i.; $r=0.5893 ; P=0.0232$; Supplemental Figure 11D). Moreover, at the latest time point on ART, the levels of activated (HLA-DR $\left.{ }^{+} \mathrm{CD} 38^{+}\right)$circulating $\mathrm{CD}^{+}(r=0.6885$; $P=0.0062$; Supplemental Figure 11E) and $\mathrm{CD}^{+}(r=0.5494$; $P=0.0339$; Supplemental Figure $11 \mathrm{~F}$ ) T cells positively correlated with residual plasma viremia. Finally, plasma levels of IP-10 (day 203 p.i.; $r=0.7575 ; P=0.0011$; Supplemental Figure 11G) and CRP (day 135 p.i.; $r=0.6986 ; P=0.0050$; Supplemental Figure $11 \mathrm{H}$ ) on ART positively correlated with SIV DNA content in intestinal tissues and residual plasma viremia, respectively. Of note, plasma viremia before ART (day 58 p.i.) negatively correlated with preART levels of intestinal Th17 $(r=-0.6988 ; P=0.0047)$ and Th22 $(r=-0.4500 ; P=0.0944)$ cells, but did not correlate with markers of immune activation on ART (Supplemental Table 3).

IL-21-treated RMs show better control of virus replication and immune activation after ART interruption. The design of the current study included a structured interruption of ART after 7 months as a tool to determine whether the reduced inflammation and virus persistence observed during ART in IL-21-treated RMs (Figures 3 and 5) would translate into superior control of viral replication and immune activation following ART interruption. In this part of the study, all SIV-infected RMs were monitored for 8 months after interruption of ART (off ART) and then euthanized (Figure 1). During the off-ART follow-up period, plasma viral loads were, on average, 0.7 logs lower in IL-21-treated RMs when compared with those in ART-only controls, although, due to animal-to-animal variability in this relatively limited cohort, the difference between the 2 groups did not reach statistical significance at any of the experimental time points (Figure 7A). The difference was partic- ularly pronounced starting at day 60 off ART, after which viral loads consistently increased (up to $1 \mathrm{log}$ ) in controls but remained stable in IL-21-treated animals (Figure 7A). To further investigate potential differences in the viral rebound after ART interruption, we then compared the levels of plasma viremia at different experimental time points off ART with the pre-ART levels. We found that plasma viremia was significantly reduced as compared with preART levels up to day 60 off ART in both groups (data not shown); however, starting at day 90 off ART, plasma viremia rebounded to levels similar to those observed before ART in control RMs, while IL-21-treated animals maintained plasma SIV RNA copies that were significantly lower than the pre-ART baseline up to day 240 off ART (Figure 7B). As expected, plasma viremia levels before ART (day 58 p.i.) strongly correlated with those after ART interruption (shown for days 90 and 240 off ART in Supplemental Figure 12), thus highlighting the critical importance of matching experimental groups for viremia before ART initiation when testing interventions, as in our study. We next measured the levels of SIV DNA in purified blood $\mathrm{CD}^{+} \mathrm{T}$ cells after ART interruption (Figure 7C) and found that, in the majority of control RMs, these levels increased as compared with their on-ART levels $(P=0.0031$; day 180 off ART), and, in fact, they were no longer different from pre-ART levels $(P=0.0829)$. In contrast, in IL-21-treated RMs, the levels of SIV DNA in blood CD4 ${ }^{+} \mathrm{T}$ cells did not increase following ART interruption ( $P=0.1931$ vs. on-ART levels $)$ and remained significantly lower $(P=0.0407)$ compared with pre-ART levels (Figure 7C). Interestingly, in 2 control animals, the levels of CD4 ${ }^{+}$ $\mathrm{T}$ cell SIV DNA content decreased after ART interruption. Both animals were $M a m u-\mathrm{A}^{*} 01^{+}$and had among the lowest set-point viral loads before ART initiation, thus suggesting the possibility of a genetically predisposed control of viral replication (38). Of note, when considering only $\mathrm{Mamu}-\mathrm{A}^{*} \mathrm{O1}^{-}$animals, we found that the levels of SIV DNA content off ART rebounded to levels significantly higher than on-ART levels in all 4 controls, but only in 1 of the 4 IL-21-treated animals (Figure 7D). As a result, CD4 $4^{+} \mathrm{T}$ cell-associated SIV DNA content on day 180 after ART interruption was significantly lower in IL-21-treated animals compared with content in control RMs (mean \pm SEM $\log _{10}: 3.11 \pm 0.52$ vs. $4.55 \pm 0.11 ; P=0.01 ;$ Figure $7 \mathrm{D})$.

We next measured, in the same SIV-infected RMs, the levels of activated or proliferating $\mathrm{T}$ cells after ART interruption. We found that IL-21-treated animals maintained $\mathrm{CD} 4^{+} \mathrm{HLA}-\mathrm{DR}^{+} \mathrm{CD} 38^{+}$and $\mathrm{CD} 8^{+} \mathrm{HLA}-\mathrm{DR}^{+} \mathrm{CD} 38^{+} \mathrm{T}$ cell levels that were significantly lower than those observed in controls in both $\mathrm{PB}$ and $\mathrm{RB}$ and up to day 240 off ART, i.e., 7 months after the last dose of IL-21 (Figure 7E). We also found that the fraction of $\mathrm{CD} 4{ }^{+} \mathrm{HLA}-\mathrm{DR}{ }^{+} \mathrm{CD} 38^{+} \mathrm{T}$ cells at necropsy was significantly lower in the LNs of IL-21-treated RMs compared with that detected in control RMs $(P=0.0260$; Supplemental Figure 13A), with a similar trend found for $\mathrm{CD}^{+}$ HLA-DR ${ }^{+} \mathrm{CD}^{2} 8^{+} \mathrm{T}$ cells (Supplemental Figure 13B). Finally, the improved control of chronic immune activation and viral replication resulted in a higher $\mathrm{CD} 4^{+} / \mathrm{CD}^{+}$ratio after ART interruption in IL-21-treated RMs, with values that become statistically significant at day 180 off ART compared with control RMs (Supplemental Figure 13C). Taken together, these data suggest that IL-21 supplementation of ART results in beneficial effects upon ART discontinuation, in particular in reducing chronic immune activation. 
A

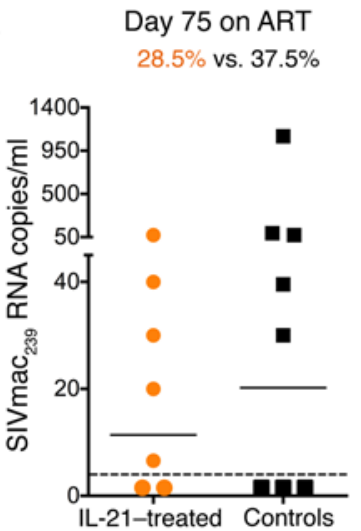

Day 104 on ART

$42.8 \%$ vs. $25 \%$

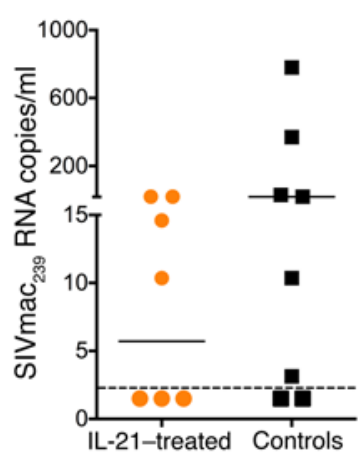

Day 200 on ART

$85.7 \%$ vs. $37.5 \%$

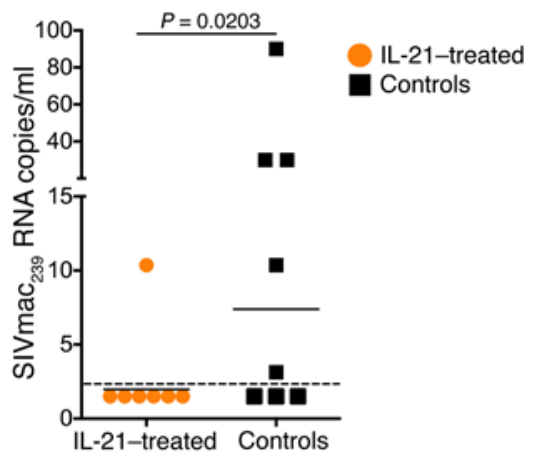

Residual viremia decrease over time is higher in IL-21-treated vs. control RMs $(P=0.03)$

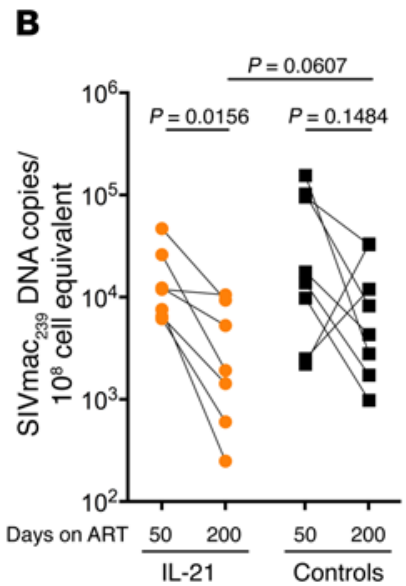

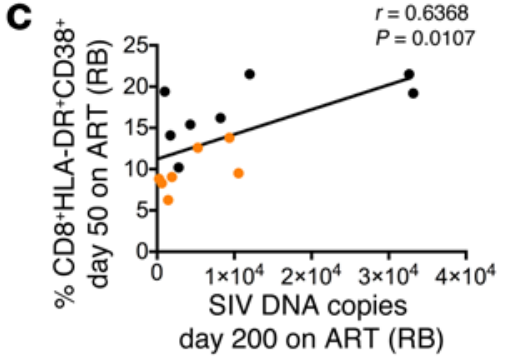

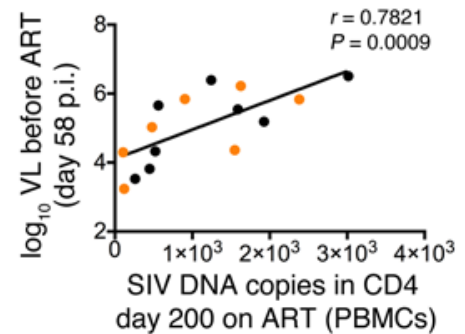

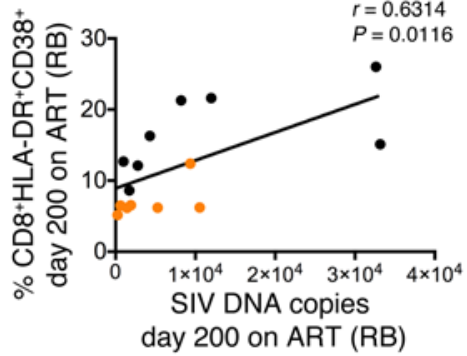

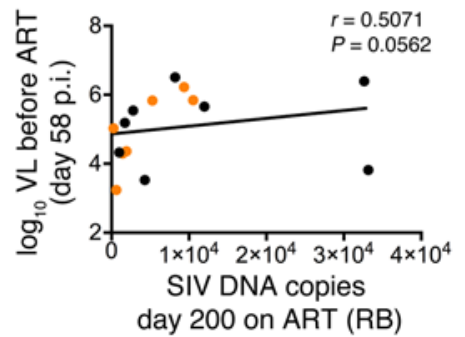

Figure 5. IL-21 supplementation reduces residual plasma viremia and SIV DNA content in ART-treated, SIV-infected RMs. (A) Residual levels (copies/ml) of plasma SIVmac ${ }_{239}$ RNA were assessed by ultrasensitive viral load assay (LOD, 3 copies per ml; dashed lines) at 3 experimental time points on ART: day 75 (day 135 p.i.), day 104 (day 164 p.i.), and day 200 (day 256 p.i.). Percentages at the top of the graphs indicate the fraction of RMs with undetectable viremia at the different time points. When all 3 time points were considered, the decrease in residual viremia was significantly higher in IL-21-treated $(n=7)$ (orange circles) RMs compared with viremia levels in controls $(n=8)$ (black squares) $(P=0.03)$. When a single experimental time point was considered, SIVmac ${ }_{239}$ RNA copies were significantly lower in IL-21-treated RMs at day 200 on ART $(P=0.0203)$. (B) Copies of total SIVmac 239 DNA per $10^{8}$ intestinal cell equivalents were longitudinally quantified at days 50 and 200 on ART. SIVmac ${ }_{239}$ DNA content significantly decreased between the 2 experimental points only in IL-21-treated animals $(P=0.0156)$. (C) Correlations between the levels of activated (HLA-DR+CD38 ${ }^{+}$CD8 ${ }^{+}$T cells at days 50 and 200 on ART and the content of SIV DNA in the rectum at day 200 on ART (top panels). Correlations between plasma viremia before ART initiation and the content of SIV DNA in PBMCs and in the rectum at day 200 on ART (bottom panels). Averaged data are presented as the geometric mean. Repeated-measures and CEE analyses were used (SAS PROC MIXED and GENMOD, version 9) to generate statistical outcomes. VL, viral load.

\section{Discussion}

While ART has a major beneficial impact on HIV disease progression, this treatment is associated with residual morbidity related to a number of persistent immune abnormalities and to the presence of a reservoir of latently infected cells that will cause a rebound of virus replication if ART is interrupted. As such, numerous concepts and products are aggressively being explored as novel interventions to be added to standard ART in order to reduce or possibly eliminate these immunological and/or virological aspects of residual HIV disease on ART. In the current study, we administered IL-21, a potent immunomodulatory cytokine, to ART-treated,
SIV-infected RMs and determined how this intervention impacted immune reconstitution, residual immune activation, and viral persistence during ART as well as after ART interruption. The rationale for using IL-21 was 4-fold. First, this cytokine regulates processes that are compromised during pathogenic HIV/SIV infections, including differentiation of Th17 cells (5-7), maintenance of functional $\mathrm{CD} 8^{+} \mathrm{T}$ cells (39-42), and differentiation of memory B cells and Ab-secreting plasma cells (43-46). Second, SIV infection is associated with a loss of IL-21-producing cells in the gut, which is correlated with the severity of intestinal Th17 cell depletion (15). Third, administration of IL-21 during acute SIV infection of RMs is 
A

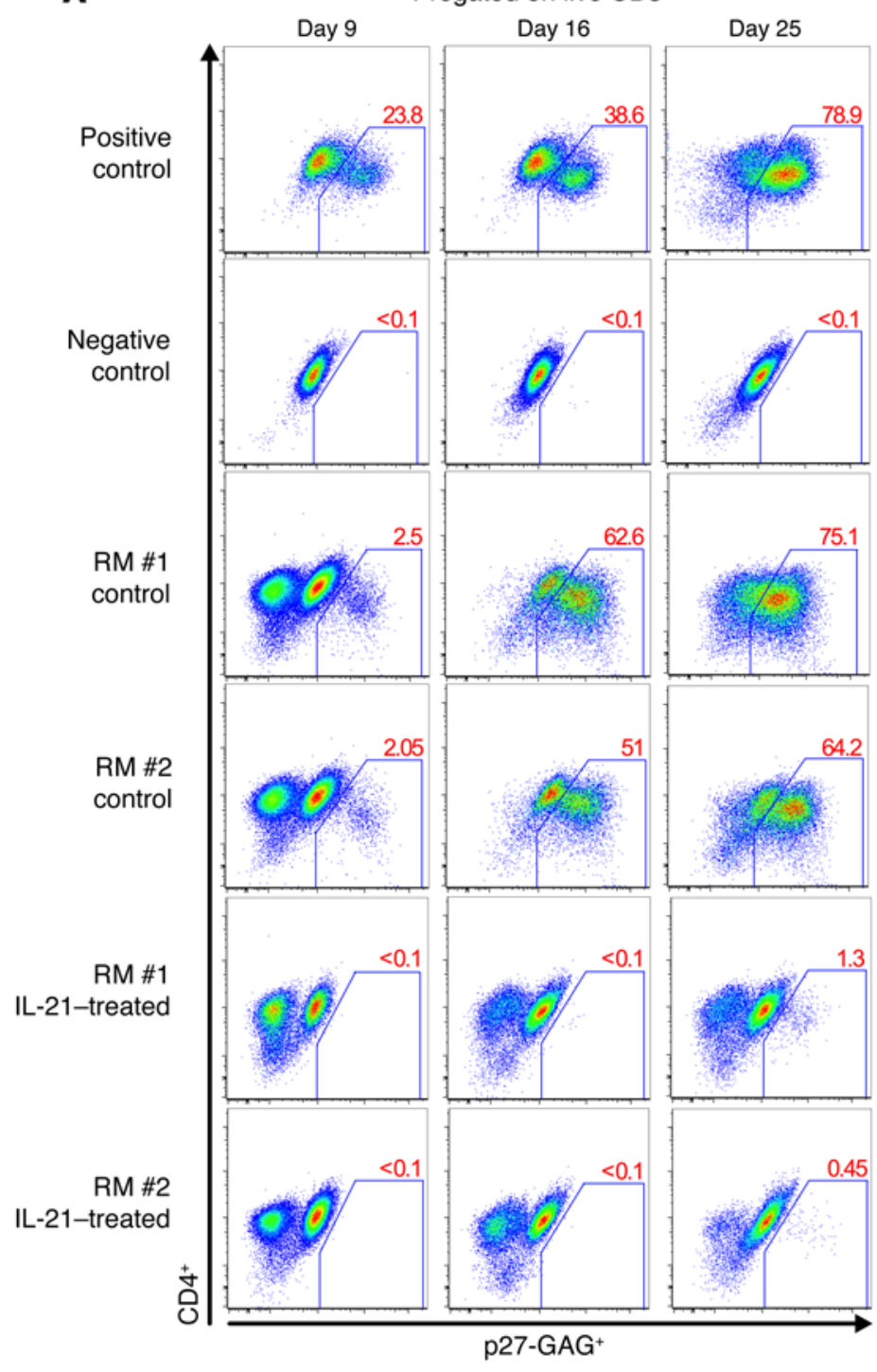

B
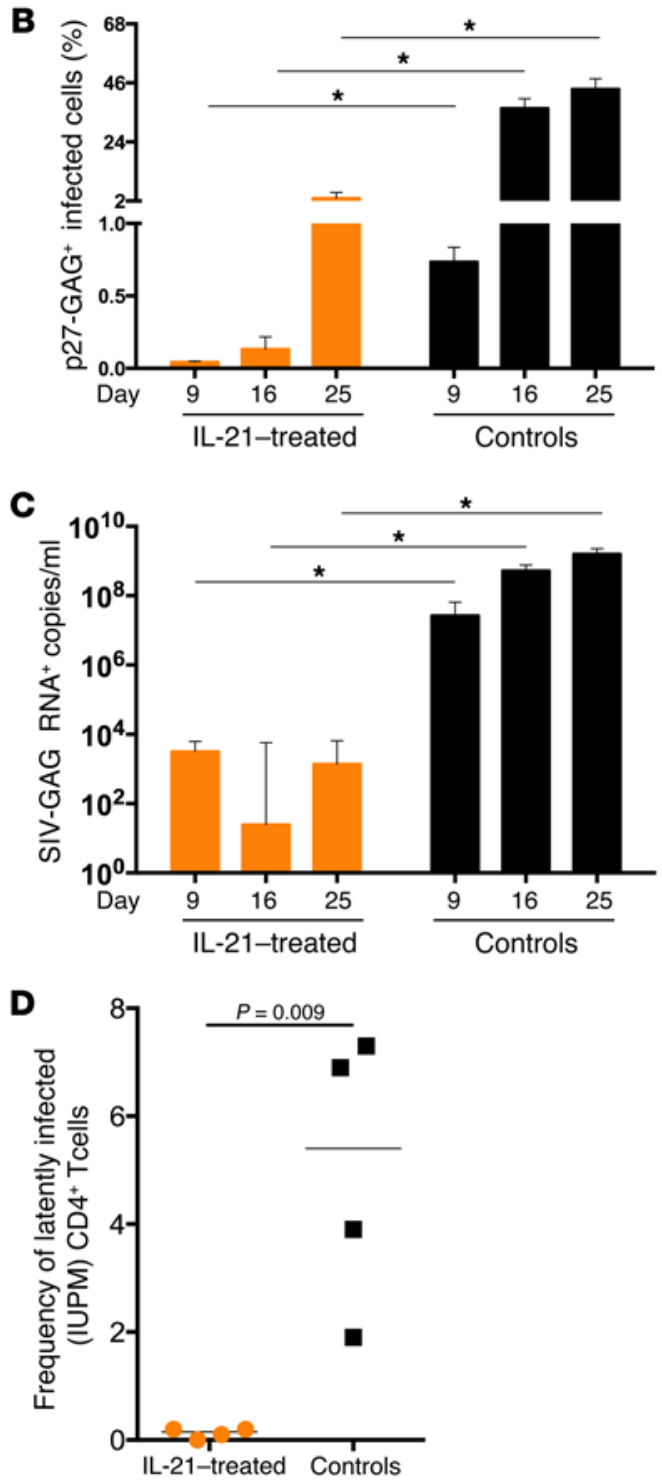

Figure 6. IL-21 supplementation reduces the frequency of $\mathrm{CD4}^{+} \mathrm{T}$ cells harboring replication-competent SIV. (A) Representative staining of flow cytometric analyses of intracellular SIV-Gag p27 on days 9, 16, and 25 in LN CD4+ T cells cocultured with CEMx174 cells. Top 2 rows show positive (CEMx174 cells infected with $\mathrm{SIVmac}_{239}$ ) and negative (CEMx174 cells, uninfected) controls. The lower 4 rows show results for 2 control (ART-only) and $2 \mathrm{IL}-21$-treated RMs. (B) Longitudinal quantification of intracellular SIV-Gag p27 and (C) supernatant SIV-Gag RNA on days 9, 16, and 25 in IL-21-treated ( $n=7$ ) (orange bars) and control $(n=8)$ (black bars) RMs. Both measurements were significantly lower $(P<0.01)$ in IL-21-treated RMs when compared with those for controls ${ }^{*} P<0.05$. (D) The frequency of latently infected cells, expressed as IUPM CD4+ $T$ cells, was significantly lower in IL-21-treated RMs (orange circles) than in control RMs (black squares) $(P=0.009)$. Averaged data are presented as the median with an interquartile range. Repeated-measures analyses were performed with a means model (SAS PROC MIXED, version 9) to generate statistical outcomes.

associated with preserved intestinal Th17 cells and limited microbial translocation (16). Last, IL-21 is currently being evaluated in several phase I and II cancer clinical trials, with limited toxicity and encouraging single-agent activity $(17,47-49)$. Importantly, a historic limitation of the SIV/RM model for studies of HIV residual disease, i.e., the lack of an optimized ART that fully suppresses virus replication, has been recently overcome, with a number of studies showing that the combination of tenofovir, emtricitabine, an integrase inhibitor (raltegravir or dolutegravir), and darunavir can durably suppress SIV infection of RMs to clinically relevant levels (50-53). Indeed, in the current study, we achieved prolonged suppression of plasma viral replication ( $<60$ SIV RNA copies/ml) in all SIV $_{\text {mac }}$-infected RMs, highlighting the relevance of the ARTtreated, SIV-infected RM model to test immune-based interventions targeting residual immune activation and virus persistence in HIV-infected humans on suppressive ART.

IL-21 administration was safe in the context of ART-treated, SIV infected RMs. When compared with ART-only controls, RMs receiving ART plus IL-21 showed (a) better reconstitution of intestinal Th17 and Th22 cells and a selective enrichment in genes that participate in Th17 and Th22 cell-orchestrated antimicrobial immunity, and (b) a significantly more rapid and pronounced reduction of the levels of cellular immune activation in rectum and blood, as well as of plasma levels of the inflammatory markers 
A

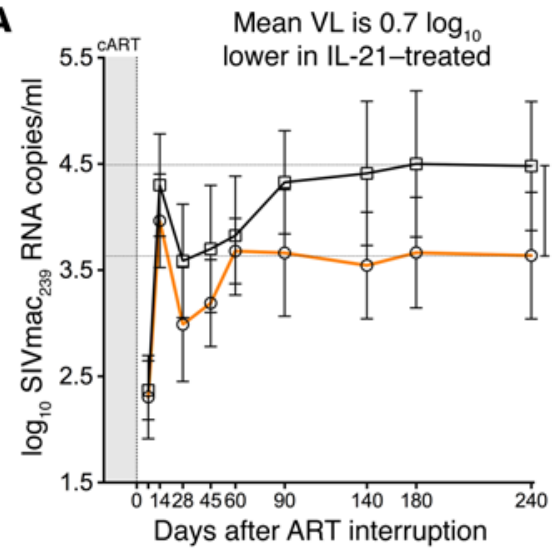

C

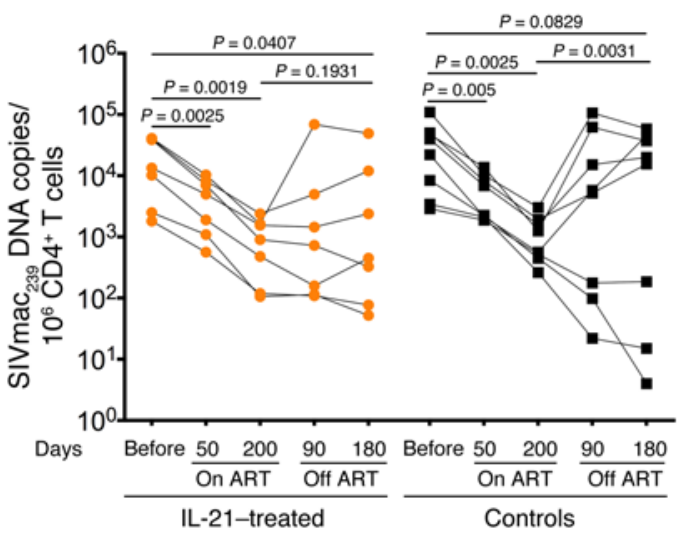

B

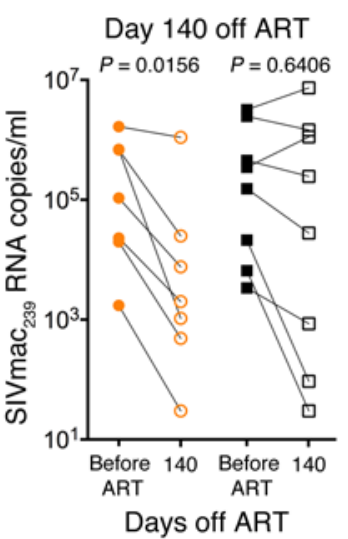

IL-21-treated

Controls

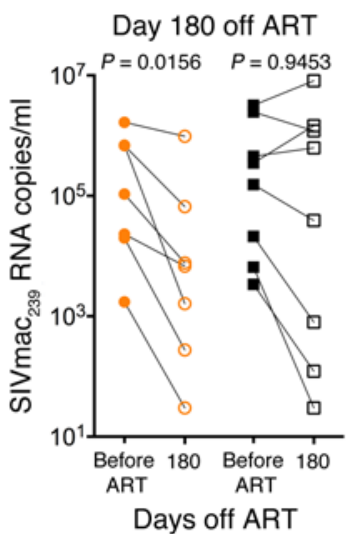

Day 240 off ART

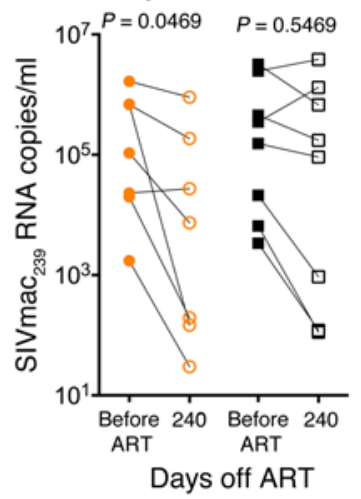

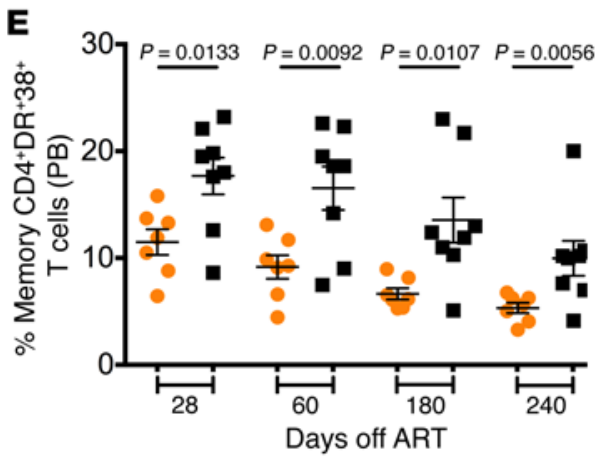

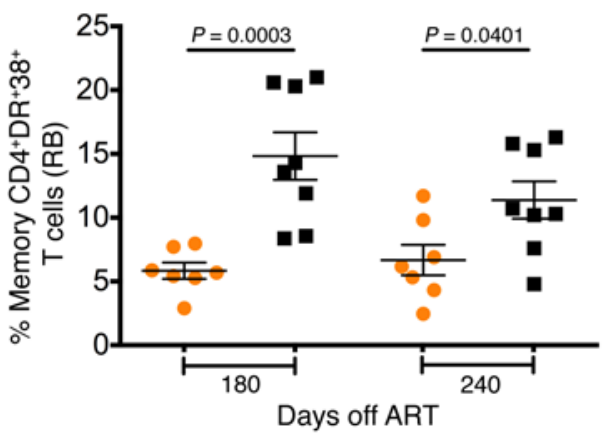

D
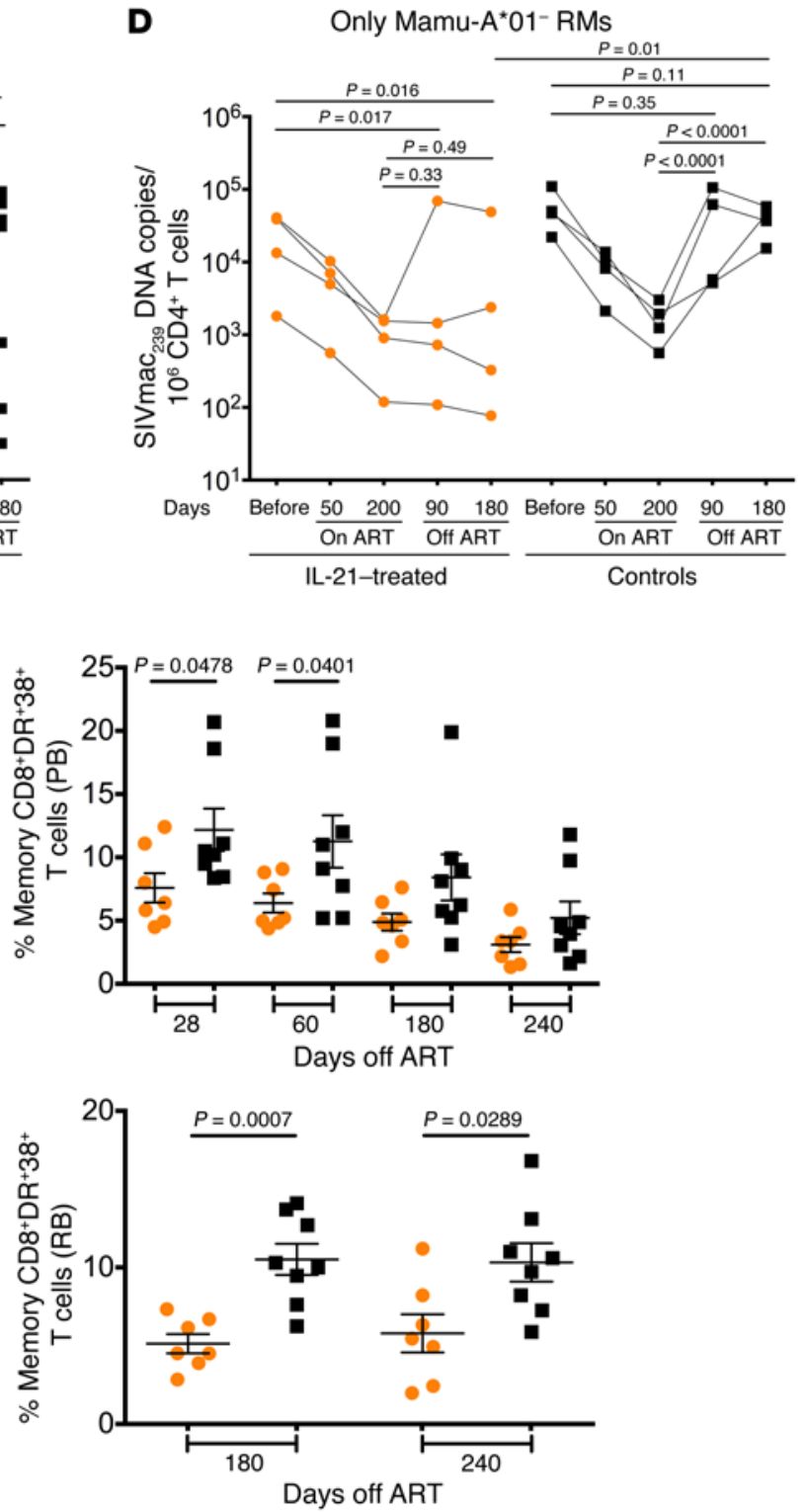
Figure 7. Effects of IL-21 treatment on immune activation and viral replication following ART interruption. (A) Longitudinal assessment of plasma $\mathrm{SIVmac}_{239}$ RNA copies (presented as the mean \pm SEM) up to 8 months after ART interruption. Starting from day 90 off ART, viremia levels were lower (average of a 0.7-log reduction) in IL-21-treated RMs up to day 240 off ART. (B) Plasma viral loads before ART (day 58 p.i.) were compared with those at days 140, 180, and 240 off ART in IL-21-treated $(n=7)$ and control $(n=8)$ RMs. At all 3 experimental time points off ART (as well as at day 90 off ART, not shown), viral loads remained significantly lower than before ART in IL-21-treated RMs but not in controls. (C) Cell-associated SIV DNA content (total) in purified blood CD4 ${ }^{+} \mathrm{T}$ cells was longitudinally determined before ART initiation (day 58 p.i.), during ART (days 50 and 200 on ART), and after ART interruption (days 90 and 180 off ART) in IL-21-treated and control RMs. At day 180 off ART, SIVmac ${ }_{239}$ DNA levels were significantly higher than levels during ART in control animals, but not in IL-21-supplemented animals. (D) Differences were even more pronounced when only Mamu- $\mathrm{A}^{*} 01^{-}$animals were analyzed. (E) Percentages of memory CD4 ${ }^{+}$ (left panels) and CD8 (right panels) T cells coexpressing the activation markers HLA-DR and CD38 were longitudinally determined at different experimental time points off ART in PB (top panels) and RB (bottom panels) biopsies. T cell activation levels were significantly lower in IL-21treated RMs compared with levels in control RMs. IL-21-treated animals are indicated with orange circles and controls with black squares. Averaged data are presented as the mean \pm SEM. Repeated-measures analyses were performed with a means model (SAS PROC MIXED, version 9) to generate statistical outcomes.

IP-10 and CRP (at selected time points). Remarkably, IL-21 treatment was associated with reduced plasma viremia, lower levels of SIV DNA in the rectum, and lower levels of replication-competent virus in $\mathrm{LN}$-derived $\mathrm{CD} 4^{+} \mathrm{T}$ cells during ART. Furthermore, IL-21treated RMs showed lower levels of immune activation and better control of virus replication up to 8 months (the latest tested experimental points) after ART interruption.

The current data in a relevant NHP model provide, for the first time to our knowledge, direct in vivo evidence of the ability of IL-21 to reduce residual immune activation during long-term ART. This is a potentially important result, since residual immune activation is considered a key contributor to non-AIDS-related morbidity and mortality in ART-treated, HIV-infected individuals $(1,3)$. More generally, this study represents, to our knowledge, the first test-ofconcept in a relevant preclinical animal model of the hypothesis that an immune-based intervention that reduces residual immune activation under ART can also limit virus persistence. Using sensitive assays, we found that the levels of SIV RNA in plasma, cellassociated SIV DNA in the rectum, and replication-competent virus in $\mathrm{LN}$-derived $\mathrm{CD}^{+} \mathrm{T}$ cells were markedly reduced at day 200 on ART in RMs also receiving IL-21. To the best of our knowledge, these are the first in vivo data directly supporting a causal link between residual immune activation and virus persistence during ART. While, on the basis of our data, we propose increased mucosal integrity as a key contributor to the reduced residual immune activation during ART in IL-21-treated RMs, the mechanisms by which a reduction of immune activation can limit virus persistence under ART may be very complex and may include (a) a reduction in the number of activated $\mathrm{CD} 4^{+} \mathrm{T}$ cells that serve as targets for the infection and that may become a persistent reservoir, and (b) a better maintenance and/or restoration of HIV/SIV-specific immune responses, thus resulting in a better clearance of productively infected cells and/or latently infected cells in which virus infection has been reactivated. Although this study was not aimed at determining the mechanism(s) of the link between residual immune activation and virus persistence, further studies that include elective necropsy of the SIV-infected RMs during ART may be able to provide critical insights on this important question. Moreover, the activity of IL-21 in increasing mucosal integrity was more pronounced when IL-21 was administered earlier during ART as compared with administration late in ART. Thus, further studies are needed to confirm the benefit of IL-21 administration on mucosal integrity in already fully suppressed SIV-infected animals. As for all therapeutic interventions improving reconstitution of $\mathrm{CD} 4^{+} \mathrm{T}$ cells during ART, one potential concern is that, by increasing Th17 cell levels, we also expand the viral reservoir harbored by these cells. In this regard, it is important to note that Th17 cell levels are never greater than those detected before SIV infection; thus, IL-21 treatment does not induce a nonphysiological accumulation of Th17 cells.

An additional and perhaps surprising result of this study is that the beneficial effects of IL-21 supplementation seem to include better control of immune activation and, although to a lower extent, viral replication following ART interruption. This observation is intriguing in that it supports the idea that immunebased interventions during ART-induced suppression of virus replication may have an impact on the dynamics of the host-virus interaction that persists even after ART discontinuation, when virus replication has rebounded to levels similar to those observed before ART initiation. The observation that IL-21 supplementation might have caused a significant and persistent reduction in immune activation despite robust virus replication is reminiscent of the phenotype typical of the nonpathogenic SIV infection of natural hosts such as the sooty mangabeys (54). Of note, in both IL-21-treated and control RMs, plasma viremia at ART initiation directly correlated with the extent of viral rebound after ART interruption, thus highlighting the critical importance of matching experimental groups before ART initiation in studies aimed at testing the curative potential of therapeutic interventions. Future studies in which the ART plus IL-21-treated, SIV-infected RMs are followed up for longer periods after ART interruption will be needed to determine the impact of this treatment on the clinical progression of SIV infection.

In summary, we tested a novel immune-based intervention in an in vivo NHP model highly relevant for studies of HIV residual immune dysfunction and virus persistence under ART. By using the well-established model of SIV infection in RMs in the context of ART-mediated suppression of virus replication, we determined that IL-21 supplementation is associated with key benefits in terms of reducing both the residual immune activation and inflammation and the level of virus persistence in ART-treated, SIV-infected RMs. In addition, these in vivo data support the hypothesis of a mechanistic link between residual immune activation and virus persistence that may have important implications in the pathogenesis of residual HIV disease under ART. Taken together, these results provide a rationale for further exploration of IL-21 as an immune-based intervention in preclinical and clinical studies of HIV/SIV eradication.

\section{Methods}

Animals, SIV infection, and ART regimen. Sixteen RMs, all housed at the YNPRC in Atlanta, Georgia, were included in the study. All ani- 
mals were $M a m u-B^{*} 08^{-}$and $-\mathrm{B}^{*} 17^{-}$, while 8 of them were $M a m u-\mathrm{A}^{*} 01^{+}$ (RLm12, RBt12, RJp11, RCb12, RVt10, RKg11, ROc10, and RPy8). The 16 RMs were randomized into 2 groups (group 1: IL-21 plus ART treatment; group 2: ART-only controls) of 8 animals on the basis of age (group 1: $6.9 \pm 0.63$ years; group 2: $6.7 \pm 0.86$ years), weight (7.4 kg \pm $0.6 \mathrm{~kg}$ vs. $6.7 \mathrm{~kg} \pm 0.3 \mathrm{~kg}$ ), and Mamu-A $\mathrm{A}^{*} 01$ status (4 in each group). All 16 animals were infected i.v. with $300 \mathrm{TCID}_{50} \operatorname{SIVmac}_{239}$ (day 0). Starting on day 60 p.i., all animals were treated with a 5-drug ART regimen consisting of 2 reverse transcriptase (RT) inhibitors (PMPA, $20 \mathrm{mg} /$ $\mathrm{kg}$ and FTC, $30 \mathrm{mg} / \mathrm{kg}$ ), 1 integrase inhibitor (raltegravir, $100 \mathrm{mg} /$ bid), and 1 protease inhibitor (darunavir, $375 \mathrm{mg} /$ bid with ritonavir, $50 \mathrm{mg} / \mathrm{bid}$, as a boosting supplementation) for 7 months. In addition, the 8 animals in group 1 received 2 cycles of treatment with recombinant IL-21-IgFc (rIL-21-IgFc) (16) $(100 \mu \mathrm{g} / \mathrm{kg}$ s.c., once per week for 6 weeks) at the beginning (from days 67 to 105 p.i.) and at the end (from days 203 to 241 p.i.) of ART, as well as 4 additional administrations upon ART interruption (day 271 p.i. or day 1 after ART discontinuation). The remaining 8 animals in group 2 served as ART-treated controls (Figure 1A). We did not notice any side effects or increased complications in the SIV-infected RMs that had been treated with IL-21 compared with those treated with ART alone. ROc10, in the IL-21-treated group, was euthanized on day 140 p.i. due to postsurgical (LN biopsy) complications. Markers in this animal were comparable to those of the others treated with IL-21, including greater Th17 and Th22 cell levels compared with those in ART-treated controls (Supplemental Table 4). On day 270 p.i., ART was interrupted, and all animals were monitored for an additional 8 months. $\mathrm{PB}, \mathrm{RB}$, and $\mathrm{LN}$ biopsies were collected at numerous experimental time points throughout the study (Figure 1).

Production and testing of rhesus rIL-21-IgFc. Rhesus rIL-21-IgFc (IL-21) fusion protein was generated as previously described by the Resource for Nonhuman Primate Immune Reagents of Emory University (16). Briefly, IL-21 was produced in the Drosophila S2 system as a fusion protein between $\mathrm{rMamuIL-21}$ and a macaque IgG2 Fc mutated to prevent binding to complement or $\mathrm{Fc}$ receptors, similar to a previously reported PD1-IgFc (55). IL-21-Fc was purified to greater than $95 \%$ by protein $\mathrm{G}$ sepharose affinity chromatography, dialyzed against PBS, and tested for sterility and the potential presence of residual endotoxin (16).

Sample collection and processing. The collection and processing of PB and LN were done as previously described $(8,15,16,56)$. Briefly, blood samples were used for a complete blood count and routine chemical analysis and plasma separated by centrifugation within 1 hour of phlebotomy. PBMCs were prepared by density gradient centrifugation. Up to $20 \mathrm{RB}$ biopsies were collected with a biopsy forceps under visual control via an anoscope. RB-derived lymphocytes were isolated by digestion with $1 \mathrm{mg} / \mathrm{ml}$ collagenase for 2 hours at $37^{\circ} \mathrm{C}$ and then passed through a $70-\mu \mathrm{m}$ cell strainer to remove residual tissue fragments. For LN biopsies, the skin over the axillary or inguinal region was clipped and surgically prepared. An incision was made over the LN, which was exposed by blunt dissection and excised over clamps. Biopsies were homogenized and passed through a $70-\mu \mathrm{m}$ cell strainer to mechanically isolate lymphocytes. All samples were processed, fixed in $1 \%$ paraformaldehyde, and analyzed within 24 hours of collection.

Flow cytometric analysis. Fourteen-parameter flow cytometric analysis was performed on PB-, PBMC-, LN-, and RB-derived cells according to standard procedures using a panel of $\mathrm{mAbs}$ that we and others have shown to be cross-reactive with RMs $(15,16,56,57)$.
Predetermined optimal concentrations of the following Abs were used: anti-CD3-APC-Cy7 (clone SP34-2), anti-CD95-PE-Cy5 (clone DX2), anti-CD28-PE-594 (clone CD28.2), anti-CCR5-APC (clone 3A9), anti-CCR5-PE (clone 3A9), anti-Ki-67-Alexa Fluor 700 (clone B56), anti-IFN- $\gamma-\mathrm{PE}-\mathrm{Cy} 7$ (clone B27), anti-CD8-PE-CF-594 (clone RPA-T8), anti-TNF- $\alpha$-Alexa Fluor 700 (clone MAb11), anti-CCR7PE-Cy7 (clone 3D12), anti-HLA-DR-PerCp-Cy5.5 (clone G46-6) (all from BD Pharmingen); anti-IL-17-Alexa Fluor 488 (clone eBio64DEC17), anti-IL-22-APC (clone IL22JOP) (all from eBioscience); anti-CD4-BV421 (clone OKT4), anti-CD4-BV605 (clone OKT4), anti-PD1-PE (clone EH12.2H7), anti-PD1-BV421 (clone EH12.2H7), anti-IL-2-BV605 (clone MQ1-17H12), anti-CD20-PerC-Cy5.5 (clone 2H7) (all from BioLegend); anti-CD8-Qdot705 (clone 3B5) and Aqua LIVE/DEAD amine dye AmCyan (both from Invitrogen); and antiCD38-FITC (clone AT-1; STEMCELL Technologies). Flow cytometric acquisition was performed on at least $100,000 \mathrm{CD}^{+} \mathrm{T}$ cells on a $\mathrm{BD}$ LSR II Flow Cytometer driven by BD FACSDiva software. Analysis of the acquired data was performed using FlowJo software.

Intracellular cytokine staining. Th17 and Th22 cell levels were determined as the percentage of $\mathrm{CD} 4^{+} \mathrm{T}$ cells that produced IL-17 and IL-22 following in vitro stimulation with PMA and ionomycin (16). PBMC-, LN-, and RB-derived cells, isolated as described above, were resuspended to $3 \times 10^{6}$ cells $/ \mathrm{ml}$ in complete RPMI 1640 medium. Cells were then incubated for 4 hours at $37^{\circ} \mathrm{C}$ in medium containing PMA, A23187, and BD GolgiStop. Following incubation, the cells were washed and stained with surface markers for 30 minutes in the dark at room temperature, followed by fixation and permeabilization. After permeabilization, cells were washed and stained intracellularly with Abs against the cytokines of interest for 1 hour in the dark at room temperature. Following staining, cells were washed, fixed in PBS containing 1\% paraformaldehyde, and acquired on a BD LSR II Flow Cytometer.

Plasma levels of immune activation and inflammation markers. Soluble IFN- $\gamma$-induced protein 10 (IP-10) and CRP levels were measured in plasma using commercially available ELISA kits according to the manufacturer's instructions. IP-10 levels were quantified using a human IP-10 Quantikine ELISA kit (R\&D Systems) and expressed as $\mathrm{pg} / \mathrm{ml}$. Plasma CRP levels were measured using a monkey CRP ELISA kit (Life Diagnostics Inc.) and expressed as $\mu \mathrm{g} / \mathrm{ml}$.

Plasma viral load and tissue-associated SIV DNA/RNA in rectum. Plasma SIV viral loads were determined by standard quantitative RT-PCR, as previously described (LOD, 60 copies/ml) (58). Ultrasensitive determinations of plasma viral loads were achieved by concentrating virus from the larger volumes of material available by ultracentrifugation as previously described (53). Quantitative assessments of SIV DNA and SIV RNA in mucosal tissues at days 50 and 200 on ART were determined by quantitative hybrid real-time/digital RT-PCR and PCR assays, as previously described (53). For each sample, 12 replicate reactions were run with a nominal single-copy sensitivity. The clinical sensitivity (based on the number of cells assessed) in our samples was as low as 1 copy/850,000 cells.

Cell-associated SIV DNA within blood CD4 ${ }^{+} T$ cells. Quantitative assessment of cell-associated total SIV DNA within circulating CD $4^{+}$ T cells at day 58 p.i., at days 50 and 200 on ART, and at days 90 and 180 off ART was performed using a modified version of a recently published quantitative nested PCR assay for cell-associated total HIV DNA (59). In a first round of PCR, total SIV DNA was amplified with 2 primers that anneal within a conserved region of the long terminal repeat 
(LTR) $5^{\prime}$ end (SIV-LF1) and at the junction with the Gag gene (SIV-R1). The forward primer SIV-LF1 was extended with a lambda phage-specific heel sequence at the $5^{\prime}$ end of the oligonucleotide. Primers targeting the CD3 gene (HCD3OUT-5' and HCD3OUT-3') were also added to quantify the exact number of cells in the initial samples. Gag-LTR sequences were amplified from $15 \mu \mathrm{l}$ lysate in a 50 - $\mu$ l reaction mixture composed of $1 \mathrm{X}$ Taq Buffer, $\mathrm{MgCl}_{2}$, dNTP, SIV-LF1, SIV-R1, and Taq polymerase. The first-round PCR cycle conditions were as follows: a denaturation step of 8 minutes at $95^{\circ} \mathrm{C}$ and then 16 cycles of amplification $\left(95^{\circ} \mathrm{C}\right.$ for 1 minute, $62^{\circ} \mathrm{C}$ for 40 seconds, $72^{\circ} \mathrm{C}$ for 1 minute), followed by an elongation step at $72^{\circ} \mathrm{C}$ for 15 minutes. In a second round of PCR, the lambda T-specific primer (Lambda T) and the LTR primer (SIV-R2) were used to amplify SIV sequences obtained from the first amplification. Primers targeting CD3 were also used in another second-round PCR. Nested PCR was performed on one-tenth of the firstround PCR product in a mixture consisting of $1 \mathrm{X}$ Rotor-Gene Master Mix (QIAGEN), Lambda T primer, SIV-R2 primers, and SIV probe (Integrated DNA Technologies). For CD3 amplification, nested PCR was performed in a mixture composed of $1 \mathrm{X}$ Rotor-Gene Master Mix, HCD3IN 5' and MamuCD3IN 3' and MamuCD3 probe. Cycling was performed on the Rotor-Gene (QIAGEN) with a denaturation step $\left(95^{\circ} \mathrm{C}\right.$ for 4 minutes), followed by 40 cycles of amplification $\left(95^{\circ} \mathrm{C}\right.$ for 3 seconds, $60^{\circ} \mathrm{C}$ for 10 seconds). The total SIV DNA copy number was calculated using a standard curve as a reference. This standard curve consisted of serial dilution of the 3D8 cell lysates (carrying 1 integrated copy of SIV genome per cell) (60).

Quantification of replication-competent virus in purified $C D 4^{+} T$ cells during ART. LN cryopreserved, highly purified $\mathrm{CD} 4^{+} \mathrm{T}$ cells were cocultured at a 1:1 ratio with the CEMx174 cell line (NIH AIDS Research and Reference Reagent Program) in serial dilutions ranging from $1 \times 10^{6}$ to $0.1 \times 10^{6}$ cells per well. The cells were cultured in complete RPMI 1640 with $4 \mathrm{mM} \mathrm{L}$-glutamine supplemented with $10 \%$ heat-inactivated FBS (Gemini Bio Products), penicillin (50 U/ml), streptomycin $(50 \mu \mathrm{g} / \mathrm{ml})$, and IL-2 (100 U/ml). Cultured cells were split, fed with fresh medium, and harvested and analyzed on days 9,16 and 25. Flow cytometric analyses of intracellular SIV-Gag p27 expression (61) and SIV-Gag viral RNA (vRNA) (copies/ml) in the supernatant were used to determine positive wells. Replication-competent SIV levels in $\mathrm{CD} 4^{+} \mathrm{T}$ cells were compared between cell cultures from controls and IL-21-treated animals. The frequencies of infected cells were determined by the maximum likelihood method (62) and expressed in terms of IUPM CD $4^{+} \mathrm{T}$ cells.

RNA-seq analysis. RNA-seq analysis was conducted at the Yerkes Nonhuman Primate Genomics Core laboratory (http://www.yerkes. emory.edu/nhp_genomics_core/). PBMCs at various time points were stored in QIAGEN RLT buffer at $-80^{\circ} \mathrm{C}$. Total RNA was prepared using QIAGEN RNeasy kits. Polyadenylated transcripts were purified on oligo-dT magnetic beads, fragmented, reverse transcribed using random hexamers, and incorporated into barcoded cDNA libraries based on the Illumina TruSeq platform. Libraries were validated by microelectrophoresis and quantified, pooled, and clustered on Illumina TruSeq v3 flow cells. Clustered flow cells were sequenced on an Illumina HiSeq 1000 in 100-base single-read reactions. RNA-seq data were analyzed by alignment and annotation to the MacaM assembly of the Indian rhesus macaque genome (63) (available at http://www. unmc.edu/rhesusgenechip/index.htm\#NewRhesusGenome).

Alignment was performed using STAR version 2.3.0e (64); parameters were set using the annotation as a splice junction reference, and unannotated, noncanonical splice junction mappings and nonunique mappings were removed from downstream analysis. Transcripts were annotated using MacaM assembly and annotation, version 7.6 (see previous URL address). RNA-seq reads and the normalized expression table were deposited in the NCBI's Gene Expression Omnibus (GEO) database (GEO GSE73232). Transcript assembly, abundance estimates, and differential expression analysis were performed using Cufflinks, version 2.1.1, and Cuffdiff (65). RNA-seq data are expressed as fragments per kilobase mapped (FPKM), which is the default output by Cufflinks/Cuffdiff, in which reads and fragments mapping to an individual gene are normalized by the total number of kilobases that map to the reference genome for an individual sample; this calculation minimizes bias due to differences in sequencing depth between samples. Heatmaps and other visualizations were generated using the Partek Genomics Suite, version 6.6.

GSEA. GSEA was performed using the desktop module available from the Broad Institute (www.broadinstitute.org/gsea/). While conventional statistics for differential gene expression determine significance on the basis of variance on a single gene, GSEA uses a cumulative statistic based on multiple genes within a biological pathway, and these multiple genes are grouped together on the basis of their common function/characteristic or empirical data (66). Gene rank was calculated for the normalized expression table using the signal-to-noise metric. The ranked dataset was screened against the Broad Institute's Molecular Signatures Database (http://www.broadinstitute.org/gsea/msigdb/ index.jsp), curated (C2) and immunologic signatures (C7) gene sets, as well as against the ISG gene set, an immune activation dataset determined using previous data from our laboratory (67). We also generated unbiased sets of genes representing IL-21-regulated genes for GSEA testing. Gene sets with $P$ values below 0.25 were considered significant according to the guidelines published on the GSEA home page.

IHC and quantitative image analysis. IHC and quantitative image analysis for the percentage of LP area that stained for $\mathrm{MPO}^{+}$neutrophils was performed as previously described $(68,69)$.

Statistics. Repeated-measures analyses for each outcome $\left(\mathrm{CD} 4^{+}\right.$ $\mathrm{T}$ cells, CD4 ${ }^{+} \mathrm{Ki}-67^{+} \mathrm{T}$ cells, Th17 cells; plasma viral load, SIV DNA within blood $\mathrm{CD} 4^{+} \mathrm{T}$ cells, etc.) were performed with a means model using SAS PROC MIXED, version 9 (SAS Institute) providing separate estimates of the means by weeks after infection and treatment groups. A compound-symmetry variance-covariance form in repeated measurements was assumed for each outcome, and robust estimates of the standard errors of parameters were used to perform statistical tests and construct 95\% CIs (70). The mean estimates and their 95\% CIs were back transformed to the original scale and reported as the geometric mean with 95\% CIs. The model-based means are unbiased with unbalanced and missing data, so long as the missing data are noninformative (missing at random). $t$ tests were used to compare the differences between the model-based treatment means (least-squares means) at each time point and to compare mean differences over time within each treatment group. Specific statistical tests were generally performed within the framework of the mixed-effects linear model. However, selected statistical tests were performed for specific data subsets. All statistical tests were 2 sided and unadjusted for multiple comparisons. A $P$ value below 0.05 indicated statistical significance. Because of the small sample sizes, results from these studies focus on the magnitude of the differences for each outcome, consistency of the findings, and biological significance. The generalized estimat- 
ing equation (GEE) approach was used to analyze the repeated binary responses (detectable or nondetectable) for residual plasma $\mathrm{SIV}_{\text {mac239 }}$ RNA (71). The binary data over time at days 75, 104, and 200 on ART were compared between the IL-21-treated and control groups by performing a GEE analysis using the SAS PROC GENMOD (SAS Institute) with an exchangeable correlation structure for the repeated data within the animal (binomial logit model).

Study approval. This study was approved by the IACUC of the YNPRC at Emory University (permit 2001973). All animal experimentations were conducted following guidelines established by the Animal Welfare Act and the NIH for the housing and care of laboratory animals and performed in accordance with institutional regulations. All efforts were made to minimize suffering of the animals.

\section{Author contributions}

LM and MP designed the research studies. LM, ESR, RF, SEB, JLH, TH, SP, CB, SG, CSM, CD, CL, SNB, MPJ, JMB, and FV conducted the experiments. LM, ESR, RF, SEB, KAE, AC, SNB, CA, JDE, JDL, MPJ, NC, and GS analyzed the data. FV and KAR provided reagents. LM, ESR, and MP wrote the manuscript.

\section{Acknowledgments}

We gratefully acknowledge Gilead (Romas Geleziunas), Johnson \& Johnson (Guenter Kraus), and Merck (Daria Hazuda) for supplying the antiretroviral drugs. The authors also thank Sherrie Jean, Stephanie Ehnert, Christopher Souder, and all the animal care and veterinary staff at the YNPRC; Barbara Cervasi and Kiran Gill at the Emory University Flow Cytometry Core; Thomas Vanderford, Benton Lawson, and Melon T. Nega at the Emory Center for AIDS Research (CFAR) Virology and Molecular Biomarkers Core;
Nirav Patel and Gregory Tharp at the Yerkes Nonhuman Primate Genomics Core; Guofu Fang and Balwan Singh at the Resource for Nonhuman Primate Immune Reagents of Emory University for producing rMamuIL-21-IgFc; and Kristina T. Ortiz of Emory University. Finally, we thank Afam Okoye and Yoshinori Fukazawa at Oregon Health \& Science University for their technical advice on the VOA. Research reported in this publication was supported by the NIAID, NIH under award numbers R01AI116379, R01AI110334, and R33AI104278 (to M. Paiardini), ORIP/OD P51OD011132 (formerly NCRR P51RR000165, to the YNPRC), ORIP/OD 5R24RR016988 and P30AI50409 (to the Emory Center for AIDS Research), as well as by amfAR grant 109109-57RGRL (to M. Paiardini). This project has been supported in part by federal funds from the National Cancer Institute, NIH, under contract HHSN261200800001E, as well as by the Division of Intramural Research, NIAID, NIH (A1001029). The content is solely the responsibility of the authors and does not necessarily represent the official views of the NIH, nor does mention of trade names, commercial products, or organizations imply endorsement by the U.S. Government.

Address correspondence to: Mirko Paiardini, Division of Microbiology and Immunology, Yerkes National Primate Research Center, Emory University School of Medicine, 954 Gatewood Road, Atlanta, Georgia 30329, USA. Phone: 404.727.9840; E-mail: mirko.paiardini@emory.edu.

Siddappa N. Byrareddy's present address is: Department of Pharmacology and Experimental Neuroscience, University of Nebraska Medical Center, Omaha, Nebraska, USA.
1. Deeks SG, Tracy R, Douek DC. Systemic effects of inflammation on health during chronic HIV infection. Immunity. 2013;39(4):633-645.

2. Siliciano JD, Siliciano RF. Recent developments in the search for a cure for HIV-1 infection: targeting the latent reservoir for HIV-1. J Allergy Clin Immunol. 2014;134(1):12-19.

3. Deeks SG, Lewin SR, Havlir DV. The end of AIDS: HIV infection as a chronic disease. Lancet. 2013;382(9903):1525-1533.

4. Katlama C, et al. Barriers to a cure for HIV: new ways to target and eradicate HIV-1 reservoirs. Lancet. 2013;381(9883):2109-2117.

5 . Yang L, et al. IL-21 and TGF- $\beta$ are required for differentiation of human $\mathrm{T}(\mathrm{H}) 17$ cells. Nature. 2008;454(7202):350-352.

6. Nurieva R, et al. Essential autocrine regulation by IL-21 in the generation of inflammatory T cells. Nature. 2007;448(7152):480-483.

7. Korn T, et al. IL-21 initiates an alternative pathway to induce proinflammatory $\mathrm{T}(\mathrm{H}) 17$ cells. Nature. 2007;448(7152):484-487.

8. Brenchley JM, et al. Differential Th17 CD4 T-cell depletion in pathogenic and nonpathogenic lentiviral infections. Blood. 2008;112(7):2826-2835.

9. Brenchley JM, et al. Microbial translocation is a cause of systemic immune activation in chronic HIV infection. Nat Med. 2006;12(12):1365-1371.

10. Estes JD, et al. Damaged intestinal epithelial integrity linked to microbial translocation in pathogenic simian immunodeficiency virus infections. PLoS Pathog. 2010;6(8):e1001052.

11. Klatt NR, et al. Loss of mucosal CD103 ${ }^{+}$DCs and $\mathrm{IL}-17^{+}$and IL-22+ lymphocytes is associated with mucosal damage in SIV infection. Mucosal Immunol. 2012;5(6):646-657.

12. Paiardini M. Th17 cells in natural SIV hosts. Curr Opin HIV AIDS. 2010;5(2):166-172.

13. Cecchinato V, et al. Altered balance between Th17 and Th1 cells at mucosal sites predicts AIDS progression in simian immunodeficiency virus-infected macaques. Mucosal Immunol. 2008;1(4):279-288.

14. Raffatellu M, et al. Simian immunodeficiency virus-induced mucosal interleukin-17 deficiency promotes Salmonella dissemination from the gut. Nat Med. 2008;14(4):421-428.

15. Micci L, et al. Paucity of IL-21-producing CD4(+) T cells is associated with Th17 cell depletion in SIV infection of rhesus macaques. Blood. 2012;120(19):3925-3935.

16. Pallikkuth S, et al. Maintenance of intestinal Th17 cells and reduced microbial translocation in SIV-infected rhesus macaques treated with interleukin (IL)-21. PLoS Pathog. 2013;9(7):e1003471.

17. Hashmi MH, Van Veldhuizen PJ. Interleukin-21: updated review of Phase I and II clinical trials in metastatic renal cell carcinoma, metastatic melanoma and relapsed/refractory indolent non-Hodgkin's lymphoma. Expert Opin Biol Ther.
2010;10(5):807-817.

18. Leone A, et al. Increased CD4 ${ }^{+} \mathrm{T}$ cell levels during IL-7 administration of antiretroviral therapy-treated simian immunodeficiency virus-positive macaques are not dependent on strong proliferative responses. Jimmunol. 2010;185(3):1650-1659.

19. Picker LJ, et al. IL-15 induces CD4 effector memory $\mathrm{T}$ cell production and tissue emigration in nonhuman primates. JClin Invest. 2006;116(6):1514-1524.

20. Villinger F, et al. IL-15 is superior to IL-2 in the generation of long-lived antigen specific memory CD4 and CD8 T cells in rhesus macaques. Vaccine. 2004;22(25-26):3510-3521.

21. White L, et al. Differential effects of IL-21 and IL-15 on perforin expression, lysosomal degranulation, and proliferation in CD8 T cells of patients with human immunodeficiency virus-1 (HIV). Blood. 2007;109(9):3873-3880.

22. Neumann B, Klippert A, Raue K, Sopper S, Stahl-Hennig C. Characterization of B and plasma cells in blood, bone marrow, and secondary lymphoid organs of rhesus macaques by multicolor flow cytometry. J Leukoc Biol. 2015;97(1):19-30.

23. Favre D, et al. Critical loss of the balance between Th17 and T regulatory cell populations in pathogenic SIV infection. PLoS Pathog. 2009;5(2):e1000295. 
24. Gordon SN, et al. Disruption of intestinal CD4 ${ }^{+}$ $\mathrm{T}$ cell homeostasis is a key marker of systemic $\mathrm{CD}^{+} \mathrm{T}$ cell activation in HIV-infected individuals. JImmunol. 2010;185(9):5169-5179.

25. Wakabayashi H, Oda H, Yamauchi K, Abe F. Lactoferrin for prevention of common viral infections. JInfect Chemother. 2014;20(11):666-671.

26. Merenstein D, et al. Colonization by Candida species of the oral and vaginal mucosa in HIVinfected and noninfected women. AIDS Res Hum Retroviruses. 2013;29(1):30-34.

27. Hernández-Santos N, Gaffen SL. Th17 cells in immunity to Candida albicans. Cell Host Microbe. 2012;11(5):425-435.

28. Landrø L, et al. Decreased serum lipocalin-2 levels in human immunodeficiency virus-infected patients: increase during highly active anti-retroviral therapy. Clin Exp Immunol. 2008;152(1):57-63.

29. Corfield AP. Mucins: a biologically relevant glycan barrier in mucosal protection. Biochim Biophys Acta. 2015;1850(1):236-252.

30. Yeaman MR, et al. Modular determinants of antimicrobial activity in platelet factor-4 family kinocidins. Biochim Biophys Acta. 2007;1768(3):609-619.

31. Radsak MP, Salih HR, Rammensee HG, Schild H. Triggering receptor expressed on myeloid cells-1 in neutrophil inflammatory responses: differential regulation of activation and survival. JImmunol. 2004;172(8):4956-4963.

32. Bostik P, Dodd GL, Villinger F, Mayne AE, Ansari AA. Dysregulation of the polo-like kinase pathway in $\mathrm{CD}^{+} \mathrm{T}$ cells is characteristic of pathogenic simian immunodeficiency virus infection. JVirol. 2004;78(3):1464-1472.

33. Hatano $\mathrm{H}$, et al. Cell-based measures of viral persistence are associated with immune activation and programmed cell death protein 1 (PD-1)-expressing CD4 ${ }^{+} \mathrm{T}$ cells. J Infect Dis. 2013;208(1):50-56.

34. Klatt NR, Chomont N, Douek DC, Deeks SG. Immune activation and HIV persistence: implications for curative approaches to HIV infection. Immunol Rev. 2013;254(1):326-342.

35. Chomont N, et al. HIV reservoir size and persistence are driven by $\mathrm{T}$ cell survival and homeostatic proliferation. Nat Med.2009;15(8):893-900.

36. Shen $\mathrm{A}$, et al. Resting $\mathrm{CD} 4^{+} \mathrm{T}$ lymphocytes but not thymocytes provide a latent viral reservoir in a simian immunodeficiency virus-Macaca nemestrina model of human immunodeficiency virus type 1-infected patients on highly active antiretroviral therapy. JVirol. 2003;77(8):4938-4949.

37. Shen A, et al. Novel pathway for induction of latent virus from resting $\mathrm{CD} 4(+) \mathrm{T}$ cells in the simian immunodeficiency virus/macaque model of human immunodeficiency virus type 1 latency. JVirol. 2007;81(4):1660-1670.

38. Micci L, et al. Virologic and immunologic correlates of viral control post-ART interruption in SIV-infected rhesus macaque. Presented at: 8th IAS Conference on HIV Pathogenesis, Treatment, and Prevention; July 19-22, 2015;
Vancouver, British Columbia, Canada. Abstract MOAA0102.

39. Barker BR, Gladstone MN, Gillard GO, Panas MW, Letvin NL. Critical role for IL-21 in both primary and memory anti-viral CD $8^{+} \mathrm{T}$-cell responses. Eur JImmunol. 2010;40(11):3085-3096.

40. Novy P, Huang X, Leonard WJ, Yang Y. Intrinsic IL-21 signaling is critical for CD8 $\mathrm{T}$ cell survival and memory formation in response to vaccinia viral infection. J Immunol. 2011;186(5):2729-2738.

41. Yi JS, Ingram JT, Zajac AJ. IL-21 deficiency influences CD8 T cell quality and recall responses following an acute viral infection. J Immunol. 2010;185(8):4835-4845.

42. Chevalier MF, et al. HIV-1-specific interleukin-21 $\mathrm{CD} 4^{+} \mathrm{T}$ cell responses contribute to durable viral control through the modulation of HIV-specific $\mathrm{CD}^{+} \mathrm{T}$ cell function. J Virol. 2011;85(2):733-741.

43. Ozaki K, et al. A critical role for IL-21 in regulating immunoglobulin production. Science. 2002;298(5598):1630-1634.

44. Ozaki K, et al. Regulation of B cell differentiation and plasma cell generation by IL-21, a novel inducer of Blimp-1 and Bcl-6. J Immunol. 2004;173(9):5361-5371.

45. Zotos D, et al. IL-21 regulates germinal center $B$ cell differentiation and proliferation through a B cell-intrinsic mechanism. J Exp Med. 2010;207(2):365-378.

46. Ettinger R, et al. IL-21 induces differentiation of human naive and memory $\mathrm{B}$ cells into antibody-secreting plasma cells. J Immunol. 2005;175(12):7867-7879.

47. Davis ID, et al. An open-label, two-arm, phase I trial of recombinant human interleukin-21 in patients with metastatic melanoma. Clin Cancer Res. 2007;13(12):3630-3636.

48. Frederiksen KS, et al. IL-21 induces in vivo immune activation of NK cells and CD8(+) T cells in patients with metastatic melanoma and renal cell carcinoma. Cancer Immunol Immunother. 2008;57(10):1439-1449.

49. Schmidt H, et al. Safety and clinical effect of subcutaneous human interleukin-21 in patients with metastatic melanoma or renal cell carcinoma: a phase I trial. Clin Cancer Res. 2010;16(21):5312-5319.

50. Shytaj IL, et al. A highly intensified ART regimen induces long-term viral suppression and restriction of the viral reservoir in a simian AIDS model. PLoS Pathog. 2012;8(6):e1002774.

51. Del Prete GQ, et al. Effect of suberoylanilide hydroxamic acid (SAHA) administration on the residual virus pool in a model of combination antiretroviral therapy-mediated suppression in SIVmac239-infected indian rhesus macaques. Antimicrob Agents Chemother. 2014;58(11):6790-6806

52. Whitney JB, et al. Rapid seeding of the viral reservoir prior to SIV viraemia in rhesus monkeys. Nature. 2014;512(7512):74-77.

53. Hansen SG, et al. Immune clearance of highly pathogenic SIV infection. Nature. 2013;502(7469):100-104
54. Chahroudi A, Bosinger SE, Vanderford TH, Paiardini M, Silvestri G. Natural SIV hosts: showing AIDS the door. Science. 2012;335(6073):1188-1193.

55 . Onlamoon N, et al. Soluble PD-1 rescues the proliferative response of simian immunodeficiency virus-specific $\mathrm{CD} 4$ and $\mathrm{CD} 8 \mathrm{~T}$ cells during chronic infection. Immunology. 2008;124(2):277-293.

56. Paiardini M, et al. Low levels of SIV infection in sooty mangabey central memory $\mathrm{CD} 4^{+} \mathrm{T}$ cells are associated with limited CCR5 expression. Nat Med. 2011;17(7):830-836.

57. Micci L, et al. CD4 depletion in SIV-infected macaques results in macrophage and microglia infection with rapid turnover of infected cells. PLoS Pathog. 2014;10(10):e1004467.

58. Amara RR, et al. Control of a mucosal challenge and prevention of AIDS by a multiprotein DNA/ MVA vaccine. Science. 2001;292(5514):69-74.

59. Vandergeeten C, et al. Cross-clade ultrasensitive PCR-based assays to measure HIV persistence in large-cohort studies. J Virol. 2014;88(21):12385-12396.

60. Nishimura Y, et al. High frequencies of resting $\mathrm{CD}^{+} \mathrm{T}$ cells containing integrated viral DNA are found in rhesus macaques during acute lentivirus infections. Proc Natl Acad Sci U S A. 2009;106(19):8015-8020.

61. Fukazawa $Y$, et al. B cell follicle sanctuary permits persistent productive simian immunodeficiency virus infection in elite controllers. Nat Med. 2015;21(2):132-139.

62. Myers LE, McQuay LJ, Hollinger FB. Dilution assay statistics. JClin Microbiol.1994;32(3):732-739.

63. Zimin AV, et al. A new rhesus macaque assembly and annotation for next-generation sequencing analyses. Biol Direct. 2014;9(1):20.

64. Dobin A, et al. STAR: ultrafast universal RNA-seq aligner. Bioinformatics. 2013;29(1):15-21.

65. Trapnell C, Hendrickson DG, Sauvageau M, Goff L, Rinn JL, Pachter L. Differential analysis of gene regulation at transcript resolution with RNA-seq. Nat Biotechnol. 2013;31(1):46-53.

66. Subramanian A, et al. Gene set enrichment analysis: a knowledge-based approach for interpreting genome-wide expression profiles. Proc Natl Acad Sci US A. 2005;102(43):15545-15550.

67. Rotger M, et al. Comparative transcriptomics of extreme phenotypes of human HIV-1 infection and SIV infection in sooty mangabey and rhesus macaque. JClin Invest. 2011;121(6):2391-2400.

68. Sereti I, et al. Decreases in colonic and systemic inflammation in chronic HIV infection after IL-7 administration. PLoS Pathog. 2014;10(1):e1003890.

69. Somsouk M, et al. Gut epithelial barrier and systemic inflammation during chronic HIV infection. AIDS. 2015;29(1):43-51.

70. Diggle PJ, Liang KY, Zeger SL. Analysis Of Longitudinal Data. 1st ed. Oxford, United Kingdom: Clarendon; 1994.

71. Zeger SL, Liang KY. Longitudinal data analysis for discrete and continuous outcomes. Biometrics. 1986;42(1):121-130. 